
\title{
28 Research Square \\ Identification of an Exosome-Related Signature Associated with Prognosis and Immune Infiltration in Breast Cancer
}

\section{Qiaonan Guo}

The Second Affiliated Hospital of Fujian Medical University

\section{Pengjun Qiu}

The Second Affiliated Hospital of Fujian Medical University

Kelun Pan

The Second Affiliated Hospital of Fujian Medical University

Jianpeng Chen

The Second Affiliated Hospital of Fujian Medical University

Jianqing Lin ( $\square$ ljq13905977336@163.com )

The Second Affiliated Hospital of Fujian Medical University

\section{Research Article}

Keywords: breast cancer, exosome, immune cell infiltration, ESTIMATE, risk model

Posted Date: January 11th, 2022

DOI: https://doi.org/10.21203/rs.3.rs-1199471/v1

License: (c) (1) This work is licensed under a Creative Commons Attribution 4.0 International License. Read Full License 


\section{Abstract}

Background: Exosomes are nanosized vesicles, play a vital role in breast cancer (BC) occurrence, development, invasion, metastasis, and drug resistance. Nevertheless, studies about exosome-related genes in breast cancer are limited. Besides, the interaction between the exosomes and tumor immune microenvironment (TIME) in BC are still unclear. Hence, we procced to study the potential prognostic value of exosome-related genes and their relationship to immune microenvironment in BC.

Methods: 121 exosome-related genes were provided by ExOBCD database and 7 final genes were selected from the intersection of 33 differential expression genes (DEGs) and 19 prognostic genes in BC. Based on the expression levels of the 7 genes, downloaded from The Cancer Genome Atlas (TCGA) database, as well as the regression coefficients, the exosome-related signature was constructed. As a result, the patients in TCGA and GEO database were separated into low- and high- risk groups, respectively. Subsequently, R clusterProfiler package was applied to identify the distinct enrichment pathways between high-risk group and low-risk group. The ESTIMATE method was used to calculate ESTIMATE Score and CIBERSORT was applied to evaluate the immune cell infiltration. Eventually, the different expression levels of immune checkpoint related genes were analyzed between the two risk groups.

Results: Results of BC prognosis vary from different risk groups. The low-risk groups were identified with higher survival rate both in TCGA and GEO cohort. The DEGs between high- and low- risk groups were found to enrich in immunity, biological processes, and inflammation pathways. The BC patients with higher ESTIMATE scores were revealed to have better overall survival (OS). Subsequently, CD8+ T cells, naive $B$ cells, CD4+ resting memory $T$ cells, monocytes, and neutrophils were upregulated, while $\mathrm{M} 0$ macrophages and M2 macrophages were downregulated in the low-risk group. At last, 4 genes reported as the targets of immune checkpoint inhibitors were further analyzed. The low-risk groups in TCGA and GEO cohorts were indicated with higher expression levels of LAG-3, CD274, TIGIT and CTLA-4.

Conclusion: According to this study, exosomes are closely associated with the prognosis and immune cell infiltration of $\mathrm{BC}$ patients. These findings may make contributions to improve immunotherapy and bring a new sight for $\mathrm{BC}$ treatment strategies.

\section{Introduction}

Breast cancer is the most frequently diagnosed malignancy among women all over the word. Its incidence is increasing every year and its mortality rate is the second highest among female malignancies[1]. Thanks to the improvements in early detection and treatment, approximately $70-80 \%$ of $\mathrm{BC}$ patients with non-metastatic disease in early stage can be cured[2]. Despite of the multidisciplinary approaches, including surgery, chemotherapy, radiotherapy, and molecular targeted therapy, advanced BC patients with distant metastases are still considered remediless[3]. In addition, resistance to endocrine therapy or chemotherapy in BC patients also poses certain challenges to reduce the mortality of them[4]. Several problems remain elusive and sufficient evidence is lacking to fully clarify the mechanisms of 
breast carcinogenesis. Therefore, it is urgent for us to have a better grasp of the potential mechanisms involved in the progression of $\mathrm{BC}$ and the effective treatments against $\mathrm{BC}$.

Recently, immune checkpoint inhibitors and biomarker-driven therapies have been validated as prospective candidates for a subset of BC treatments[5]. Substantial inflammatory cells were reported to infiltrate in BC, which were observed not only around the tumor but also abundantly in the tumor stroma[6]. Some previous studies indicated that the interrelationship between CD8+ T cells and immune escape was close. Besides, the infiltration of macrophages, antigen-presenting cells (APCs), CD4+ T cells, dendritic cells (DCs), and other tumor-infiltrating immune cells was significantly correlated with the BC prognosis[7-9]. Immunotherapy has recently made significant advances in the field of antitumor therapy. Based on immune modulation between the cancer cells and the tumor microenvironment (TME), immunotherapy offered clinical benefits over conventional treatments via sustained anti-tumor immune responses stimulated[10]. The two non-tumor components in the TME are mainly stromal and immune cells. Hence, the aberrant gene expression generated by epigenetic alterations in TME cells were proposed to predict the clinical outcomes of tumors[11].

In 1985, exosomes were first reported in incubation of sheep reticulocytes, produced from intracellular vesicles membrane by means of budding[12]. After that, exosomes were identified released from several cell types, including but not limited to endothelial cells, immune cells, and tumor cells[13]. As a type of homogenous membrane vesicles, exosomes can be collected in body fluids with an average size of 40150nm, for instance, serum, saliva, urine, and cerebrospinal fluid[14]. Later studies further demonstrated that exosomes played a vital role in intercellular communication and molecular transfer, containing celltype specific exosomal proteins, lipids, and nucleic acids, as well[15]. In the interaction of cancer cells and their surrounding microenvironment, exosomes were involved in shaping the tumor immune responses by targeting MDSC, CAF, TAM, immune suppressive Treg cells and so on[16-18]. Recently, exosomes have been reported involved in various cancer progression[19-21]. In breast cancer, exosomes were shown to deliver mRNAs that could lead to tumor information transformation in non-tumorigenic cells[22]. In melanoma, exosomes were found to have a relationship with pre-metastatic niche formation, which could transfer MET to bone marrow progenitor cells[23]. Interestingly, several studies shown that a portion of the human epidermal growth factor receptor (HER) family was associated with exosomes in BC, gastric cancer and pancreatic cancer[19-21]. Hence, understanding the certain functions of exosomes in the TME can further provide novel sight in exosome-based biomarkers and immunotherapy for tumor diagnosis and treatment.

The aim of this study was to investigate the relationship between exosome-related genes and the TIME in breast cancer. The gene profile data of BC patients were downloaded from the TCGA (http://cancergenome.nih.gov/) and GEO database (https://www.ncbi.nlm.nih.gov/geo/). After that, exosome-associated genetic data were extracted from ExOBCD database[24] to analyze to establish an exosome risk model. Subsequently, the prognosis of $\mathrm{BC}$ patients was predicted according to the exosome-related risk model. Furthermore, as an entry point, the exosome-related risk score was adopted to identity the distinction in immune cell infiltration rate. Accordingly, we searched further for correlations 
between exosome-related risk scores and TIME, and investigated 4 genes previously reported to be associated with immune checkpoint blockade (ICB) as well[25]. This important therapy will be used to develop various interesting combination treatment strategies in the future.

\section{Materials And Methods}

\subsection{Workflow}

A combination of several methods was applied to construct a 7-exosome-related-gene signature and explore the potential mechanisms by which these genes affect the prognosis of breast cancer (Figure 1).

\subsection{Data acquisition}

RNA-sequencing expression data and clinical information of breast cancer patients were extracted from TCGA database and the corresponding information from GSE20685 was collected from GEO database as the training set and the validation set, respectively. After batch normalization, part of patients was excluded because of incomplete clinical data and an OS of less than 90 days. With complete information 828 BC samples from TCGA database were included as training set and 327 BC samples from GEO database were included as validation set for subsequent analysis. In addition, 121 exosome-related genes collected from ExoBCD database were provided in Supplementary Table1.

\subsection{Construction of a risk model}

The exosome-related independent prognostic genes were screened via Cox regression univariate analysis of OS and presented by Forest plots. The DEGs between BC samples and normal breast tissue samples in training set were analyzed by edger package. The genes that met cutoff criteria of |log2fold change (FC) $\mid>1$ and $p$-value $<0.05$ were considered as DEGs, visualized by volcano plot. Afterward, the overlapping exosome-related genes of DEGs and prognostic genes were screened out via drawing Venn diagrams for subsequent analysis. In order to reduce redundant genes and obviate model overfitting, the least absolute shrinkage and selection operator (LASSO) Cox regression model was established to determine all independent prognostic genes[26]. The exosome-related genes expression levels were used

to construct the risk score formula as: Risk score $=\sum_{i=1}^{n}\left(\operatorname{Exp}_{i}{ }^{*} \operatorname{Coe}_{i}\right) .\left(\mathrm{N}=7, \operatorname{Exp}_{i}\right.$ represented the expression level of each selected gene, and $\mathrm{Coe}_{i}$ denoted the corresponding Cox regression coefficient.) According to the median risk score, patients with breast cancer were separated into low- and high- risk groups. Survival analyses were carried out in different risk groups by means of the "survminer" $\mathrm{R}$ package. Time-dependent ROC curve analyses was further performed to assess the predictor efficacy of this gene signature. As consequence, univariate and multivariate COX regression analyses were conducted to validate that the exosome-related risk score was an independent factor of prognosis for patients suffering from breast carcinoma. The hazard ratio (HR) was calculated to identify the $95 \%$ confidence interval. Hierarchical clustering was adopted to analyze the relationship between expression 
levels of 7 selected genes and molecular characteristics as well as clinical features, presented by heat map.

\subsection{Functional enrichment analysis}

Gene Ontology (GO) enrichment analyses and Kyoto Encyclopedia of Genes and Genomes (KEGG) pathway analyses were conducted for DEGs between high-risk groups and low-risk groups with the $\mathrm{R}$ clusterProfiler package. Biological process (BP), cellular component (CC), and molecular function (MF) are involved in GO terms. P values < 0.05 were considered significant in functional enrichment analysis.

\subsection{Assessment of the tumor immune microenvironment}

Estimation of Stromal and Immune cells in Malignant Tumor tissues using expression (ESTIMATE) algorithm consist of Immune Score, Stromal Score and ESTIMATE Score[27]. The proportion of the immune-stromal component of TME was calculated by "estimate" R package. The respective scores implied the ratio of the corresponding compositions in the TME.

\subsection{Evaluation of immune cell type components}

As a commonly used method for estimating and analyzing immune cells infiltration, CIBERSORT (http://cibersort.stanford.edu/) was used to evaluate the proportion of distinct cell subtypes from mixed cell specimens by RNA-seq expression profile. 22 marked immune cell subtypes are consist of 7 types of T cells, naive and memory B cells, plasma cells and myeloid subsets and LM22 is usually used to present the annotated gene expression signatures. Therefore, CIBERSORT was employed to calculate the ratios of 22 marked immune cell subtypes among different risk groups[28]. The assumption of immune cell types was accurate and statistics at $\mathrm{P}<0.05$ were used for further analysis. Eventually, the ratios of tumor immune infiltrating cell (TIIC) types for each tumor specimen were assessed, as well. The Wilcoxon test was carried out to characterize TIIC between tissues in different exosome-related risk groups.

\subsection{Correlations between immune checkpoint genes and exosome-related risk score}

The genes reported to play significant roles in immune responses were collected. Afterwards, the GGPUBR, ggplot2, and ggExtra packages of R software were used to determine the associations of gene expression levels with different exosome-related risk scores.

\subsection{Statistical analysis}

All statistics analysis were performed via R software (Version 4.0.5) (https://www.r-project. org/). Wilcoxon test was employed to examine the differences between variables of two groups. The survival data was assessed by Kaplan-Meier curve. The univariate and multivariate Cox regression analysis were employed to identify the independent prognostic factors. $\mathrm{P}<0.05$ was regarded as of a statistical significance.

\section{Results}




\subsection{Identification of 7 exosome associated DEGs in the TCGA cohort with prognostic relevance}

The exosome-related gene set was downloaded from ExoBCD database, containing 121 genes participate in exosome-related regulation. Among them, 117 genes were ascertained in TCGA cohort. Subsequently, 19 exosome-related genes were identified to associate with BC patients' OS via univariate Cox regression analysis $(P<0.05)$, presented by forest plot in Figure 2A. Afterwards, 112 normal breast tissues and 828 breast carcinoma tissues were enrolled in this study, resulting that 33 overlapping DEGs were identified by edger package (Figure 2B). Consequently, 7 overlapping exosome-related genes were selected from DEGs and independent prognostic genes via Venn diagrams (Figure $2 \mathrm{C}$ ).

\subsection{Construction and validation of a gene-based prognostic model}

To avoid the elimination of significant prognostic genes, 7 genes stated above were subjected to LASSO regression analysis. LASSO coefficient overview of 7 selected genes were shown in Figure $3 \mathrm{~A}$ and 10 -fold cross-validation outcome was generated to confirm the preferred value of the penalty parameter $\lambda(\lambda=$ 0.0009570788) (Figure 3B). The 7 vital genes were EPCAM, PIGR, KRT14, DOK7, CD24, CYP19A1, and CXCL13. Furthermore, a prognostic risk model for evaluating BC patients' OS was established based on the expression levels of the 7 vital genes stated above and their regression coefficients as described below: Risk score $=(0.153 \times$ expression level of EPCAM $)+(-0.042 \times$ expression level of PIGR $)+(-0.040 \times$ expression level of KRT14) $+(-0.069 \times$ expression level of DOK7 $)+(0.154 \times$ expression level of CD24 $)+$ (0.105x expression level of CYP19A1) $+(-0.106 \times$ expression level of CXCL13). Afterwards, the patients were separated into high- and low- risk groups based on the median risk score (Figure 4C).

In Figure 4A, the Kaplan-Meier curves suggested that patients with high-risk score are indicated worse survival rates in the training set $(P<0.01)$. After that, the time-dependent ROC analysis was conducted at 2,3 and 5 years to assess the predictive efficacy of this risk model. As consequence, the prognostic features identified were verified robust efficient in predicting $O S$ in $B C$ patients via the area under the curve (AUC) (AUC=0.705, 0.742 and 0.669; at 2,3 and 5 years, respectively, Figure 4B). Similarly, 327 patients of GSE20685 were enrolled as the validation set and the risk score for each sample was work out based on the exosome-related signature mentioned. Figure 4E shows that patients with high-risk score are indicated worse survival rates in validation set according to the Kaplan-Meier curves $(P<0.01)$. Remarkably, the risk model had been shown to have promising long-term prognostic predictive efficacy, as reflected in the time-dependent ROC analysis (AUC=0.727, 0.691 and 0.695; at 2,3 and 5 years, respectively, Figure $4 \mathrm{~F}$ ). Hence, the exosome-related genes signature to evaluate $\mathrm{BC}$ patient's prognosis was successfully constructed. Subsequently, based on the median risk score, the BC patients in training and validation sets were separated as high-risk groups and low-risk groups (Figure $4 \mathrm{C}$ and $4 \mathrm{G}$ ). As shown in Figure 4D and $4 \mathrm{H}$, patients with higher risk scores were manifested poorer prognosis whereas patients with lower risk scores were manifested better prognosis. 
To further assess the efficacy of the 7-gene signature predicting the prognosis of BC patients as an independent factor, the 7-gene signature along with some covariates, for instance, tumor stage, ER, PR, HER2 and age were subjected to the univariate and multivariate Cox regression analysis. Consequently, the results revealed that the exosome-related risk score was an independent prognostic factor for $\mathrm{BC}$ patients in TCGA cohorts (Figure 5A and B), also in GEO cohorts (Figure 5D and E). Heat maps were applied to show clinical characteristics, molecular features, and distinct expression levels of 7 screened genes through hierarchical clustering in training set (Figure 5C) and validation set (Figure 5F).

\subsection{GO and KEGG functional enrichment analysis}

GO and KEGG analysis were performed in the DEGs between high and low risk groups to identify the signaling pathways and biological functions associated with exosome-related risk score. As a result, the first $30 \mathrm{GO}$ terms including CC, BP and MF were displayed in Figure 6. Additionally, 10 and 30 enriched KEGG pathways in training and validation set were manifested in Figure 7, respectively. Most of these GO terms and the KEGG pathways were associated with immune activation as well as biological functions.

\subsection{Correlation of ESTIMATE score and 7-gene signature}

The ESTIMATE algorithm was applied to derive an ESTIMATE fraction for each sample, indicating the overall extent of immune infiltration and the TME landscape. As presented in Figure 8, in both cohorts, stromal scores, immune scores, and ESTIMATE scores were proved higher in low-risk groups rather than high-risk groups $(P<0.05)$. Consequently, combined with the result showed in Figure $4 A$ and $E$, the high stromal, immune and ESTIMATE scores manifested a better OS, on the other hand, the low scores were associated with the poor OS.

\subsection{Distribution of infiltrative immune cells in breast cancer}

The functional enrichment analysis revealed that the DEGs between high-risk and low-risk groups were generally enriched in the pathways related to inflammation, immune responses, etc. Therefore, the CIBERSORT algorithm was adopted to obtain the TIIC ratios and to construct TIIC profiles. Figure $9 \mathrm{~A}$ and $B$ show the proportions of infiltrating immune cells in training set and validation set, respectively. As presented in Figure 10A and B, naive B cells $(P<0.001), C D 8+T$ cells $(P<0.001), C D 4+$ resting memory $T$ cells $(P<0.001)$, and Monocytes $(P<0.001)$ were upregulated, whereas M0 macrophages $(P<0.001)$ were downregulated in low-risk group of training cohort. As for validation cohort, Neutrophils $(P<0.001)$ were upregulated, while M2 macrophages $(P<0.001)$ were downregulated in low-risk group. Consequently, the study targeting exosome-related genes could be a groundbreaking finding for the immunotherapy of tumor sufferers in the future.

\subsection{The relationship between exosome-related risk model and immune checkpoint genes}

The expression levels of immune checkpoint genes associated with the therapeutic response to immune checkpoint inhibitors were investigated. PD-L1(CD274), LAG3, CTLA4 and TIGIT were previously reported to be the targets of immune checkpoint inhibitors. As shown in Figure 11, the expression levels of 4 
mentioned genes were higher in low-risk group in TCGA cohorts. Likewise, in GEO cohorts, the expressing levels of them were upregulated in low-risk group statistically with $P<0.01$, except CTLA4 $(P>0.05)$.

\section{Discussion}

Breast cancer is the highest incidence and deadliest type of carcinoma for women worldwide, presenting highly heterogeneous biological and clinical characteristics[29]. As a kind of nanosized vesicles, exosomes play vital roles in tumor development and progression. Importantly, they can regulate cell-tocell communication in the tumor microenvironment via proteins, lipids and RNA cargo transferred[30]. Most recently, ICB therapy has made promising progress in cancer immunotherapy. In 2017, Chen and colleagues raised the idea that tumors with lower PD-L1 expression level and fewer infiltrating cytotoxic T cells were considered as immune "cold" tumors, where ICB therapy achieved only limited results. Fatally, "cold" tumors account for the majority[31]. Increasing evidence suggested that exosomes had the potentiality to work as biomarkers for a variety of malignant tumors including breast carcinoma. Previous study in pancreatic cancer identified that exosomes released from tumor contained a membrane bound protein called GPC1, considered as a sensitive and unique biomarker in early-stage disease[32]. Interestingly, a study in breast cancer indicated that the expression level of serum exosomal-annexin A2 (exo-AnxA2) could be detected higher in women with carcinoma compared with non-cancer, particularly for triple-negative breast cancer (TNBC) rather than luminal or HER2-positive breast cancer[33]. These results suggested that exosomes played an important role in $\mathrm{BC}$ progression and the possibility to construct a prognostic model by these exosome-related genes. In the current study, we adopted bioinformatics analysis to examine changes in the expression profiles of 121 exosome-related genes in breast cancer and the relationship with OS. Among them, 7 exosome-related genes were identified to establish a novel prognostic signature. Subsequently, the BC patients in TCGA and GEO cohorts were divided into high and low risk groups respectively according to the prognostic signature. Moreover, the DEGs between the high-risk group and the low-risk group were identified and the functional analysis were further performed, proposing that immune-related biological processes were highly enriched. Eventually, the infiltration ratios of distinct immune cells in breast cancer samples were analyzed. The results revealed that the groups at high risk of exosomes were immunologically 'cold', while the groups at low risk were immunologically 'hot'.

The 7 prognostic genes associated with exosomes consist of EPCAM, PIGR, KRT14, DOK7, CD24, CYP19A1, and CXCL13. As one of the first tumor-related antigens, epithelial cell adhesion/activating molecule (EpCAM/CD326) has been reported highly overexpressed in primary and metastatic breast cancer, leading to poor prognosis[34]. Furthermore, study in hepatocellular carcinoma (HCC) suggested that tumor growth and invasion were associated with EpCAM-positive cells, which was one of the components of targeting Wnt/beta-catenin signaling pathway[35]. Cluster of differentiation 24 (CD24) is a glycosyl-phosphatidyl-inositol (GPI)-anchored glycoprotein, that has been demonstrated as a vital role in multiple areas. In cancer, CD24 is highly expressed in various tumor cells, including breast cancer cells, and associated with the growth, invasion, and migration of tumor cells[36-40]. In immunology, as a primarily costimulatory molecule, CD24 can achieve effective immunosuppression and tumor immune 
escape via activating a series of intracellular signal pathways and regulating multiple immune cells, for instance, T cells, B cells, macrophages, and NK cells[41]. Notably, Barkal and colleagues revealed that the macrophages enhanced their capability to engulf tumors and slowed down the development of macrophage-dependent tumors in vivo through gene knockout to blocking CD24 and Siglec-10[42]. This is consistent with our conclusion, the infiltration level of $\mathrm{MO}$ macrophages is higher in high-risk group. Polymeric immunoglobulin receptor (PIGR) is a critical element of the mucosal immune system and intermediates epithelial cell transfection of immunoglobulins. And the expression level of PIGR was demonstrated decreased in nasopharyngeal carcinoma cells by Qi and colleagues, related to poor prognosis $[43,44]$. Several studies suggested DOK7 as a potential tumor-suppressor gene, because of the significantly low expression levels in BC tissues compared with normal tissues. The lower expression level of DOK7 was associated with the greater aggressive clinical behaviors and poorer prognosis in $\mathrm{BC}[45]$. The mechanistic studies by Yue et al. illustrated that DOK7 inhibited proliferation and invasion of BC cells through PI3K/PTEN/AKT pathway[46]. CXCL13 may be another potential protective factor for BC patients' prognosis. Although, CXCL13 has been reported in many types of carcinomas to drive signaling pathways associated with proliferation and invasive, including PI3K/AKT pathway[47] and Wnt/betacatenin signaling pathway[48], in patients with HER2-positive BC or TNBC, increased CXCL13 corresponded with better survival[49]. In addition, CXCL13 has been shown to increase B cell and T cell infiltration in multiple tumor types and associate with greater prognosis and survival[50, 51].

In our study, the results of functional enrichment analysis indicated that many GO terms and KEGG pathways were associated with biological processes and immune cells activation. In 1996, immunologists were interested in the relationship between exosomes and cells from immune system. They found that Epstein-Barr virus-transformed B lymphocytes could secrete exosomes by fusion of MVBs with the plasma membrane[52]. Furthermore, some discoveries suggested that exosomes played key roles in adaptive immune responses. Exosomes released from these kinds of cells harbor MHC class II dimers bound to antigenic peptides and the exosomes were indicated to present the MHC-peptide complexes to specific T cells. In addition, dendritic cells (DCs) in mice were reported to secrete exosomes with functional MHC class I-peptide complexes, which could improve the triggering of CD8+ Tlymphocyte-dependent immune responses[53,54]. Recently, exosomes have been shown to be involved in promoting immune responses. Studies in human pancreatic and colorectal tumours conducted by Gastpar R and his colleagues suggested that NK cells cultured with tumour-derived Hsp70-positive exosomes were induced to liberate granzyme B that activated apoptosis[55]. As the mechanisms of exosomes in antigen-specific immune responses were better understood, several studies showed that tumor-released exosomes also carried a variety of immunosuppressive molecules, such as CD8 and CD4 T lymphocytes[56, 57], NK cells[58], regulatory T lymphocytes[59] and myeloid cells[60]. Hence, we reasonably assumed that exosomes were closely associated with anti-tumor immunity in BC. Furtherly, The CIBERSORT algorithm was applied to derive the ratios of various kinds of tumor-infiltrating immune cells to reveal the relationship between exosomes and immune cell infiltration in BC. The result indicated that the infiltrated tumor-killing immune cells were significantly reduced in high-risk group instead of lowrisk group, such as CD8+ T cells and activated NK cells. However, the immune cells that promoted tumor 
progression and migration, M2 macrophages and M0 macrophages, were reduced in low-risk group instead of high-risk group. As a marker of tumor progression and drug resistance, TIME was characterized by tumor inflammation promoting and tumor cells immune surveillance[61]. Recent studies in tumors observed that some immune activities were associated with exosomes. Studies in exosomes secreted from prostate cancer cells indicated that exosomes contained ligands for natural killer group 2D (NKG2D) could downregulate the expression of NKG2D on NK cells and impair the cytotoxicity of NK cells[58, 62]. Besides, those exosomes secreted by HCC cells with chemotherapy treatment efficiently stimulated NK cells to product the granzyme B, hence promoted the tumoricidal function[63]. In tumor microenvironment, those tumor cell-derived exosomes could influence the DCs status. The results of experiments in vitro indicated that exosomes produced by TS/A BC cells could block the differentiation progression of DC from myeloid cells[64]. In terms of macrophages, type 1 macrophages (M1) were involved in anti-tumor immune responses by acting as antigen-presenting cells whereas type 2 macrophages (M2) acted in the pro-tumor immunity as the common phenotype of tumor-related macrophages[15]. Several studies revealed that the M2 status was correlated with the progression of tumors and poor prognostic outcomes of patients. Yang $\mathrm{M}$ et al. reported that exosomes derived from M2 could promote BC cell growth and invasion by transferring miR-223[65]. Additionally, neutrophils status was reported to be regulated and differentiated via the stimulation from TME, as a result, to further moderate tumor immune responses and regulate tumor progression. Bobrie $A$ and colleagues investigated on the value to Rab27a in exosome secreted by BC cells, consequently, exosomes were identified to induce systemic mobilization to neutrophils to facilitate tumor progression[66]. Not only different kinds of immune cells, but also other cellular components of TME, including MSCs, endothelial cells and fibroblasts, played significant roles in tumor progression[15]. Accordingly, a conclusion was raised that the exosomes were markedly associated with the ratio of tumor-related immune cells infiltrating in $\mathrm{BC}$, and the low-risk cohort tended to possess higher ratio of cytotoxic lymphocyte infiltration.

Recent research findings suggested that exosomes were able to control the core immunologic processes and regulate inflammatory response. Immune cell exosomes were identified to involve in stem cell mobilization, immunological regulation, and tissue remodeling[67]. Immunotherapy is increasingly becoming the key to BC treatment. Yang $Y$ et al. found that PD-L1 could be carried by BC cell-derived exosomes and transferred to tumor cells expressed low levels of PD-L1 to blocked T cell activity[68]. Notably, exosome-related immunotherapeutic exoPDL1 was able to be applied to develop novel drugs with minimum toxicity and considerable clinical effectiveness[67]. Furthermore, immune checkpoints, a key of facilitating tumor immunosuppression, were analyzed in different risk groups to detect the correlation between exosomes and immune checkpoint inhibitors. Stimulation of immune checkpoint targets can block tumor attack. In our study, high-risk group predicated on 7-exosome-related gene risk model was found to associate with lower expression levels of immune checkpoint genes and poor clinical outcomes. As a result, mammary tumor in high-risk group were considered immunologically "cold" and difficult to gain benefits from ICBs. However, the tumors in low-risk group were considered 
immunologically "hot" and more possible to get benefits from ICBs[31]. Accordingly, the exosome-related risk model had highly potential to predict the efficacy of ICBs in treatment for patients suffered from BC.

It was the first study to construct an exosome-related risk model for breast cancer based on 7 exosomerelated genes by use of public databases with retrospective data. This risk model can serve as an independent prognostic factor in BC patients. In addition, some limitations in current research should be noticed. Some of the clinical data in the TCGA or GEO cohorts were incomplete and the absent data may not be random, leading to the bias in the analysis of clinical relevance. Therefore, it requires more extensive multi-center clinical validation to further back up our ideas. Besides, this prognostic model was built only from exosome-related genes and various other hotspot biomarkers were not enrolled.

Consequently, further experimental validation of the relationship between immune cells and exosomes is needed to provide new perspectives in immunotherapy and tumor treatment.

\section{Conclusion}

In summary, by combining bioinformatics tools and related algorithms, we identified a novel exosomerelated risk model associated with immune infiltration. It can be served as a potential independent prognostic factor and bring new insights into anti-tumor immunity for breast cancer.

\section{Declarations}

\section{Ethics approval and consent to participate}

No applicable.

\section{Consent for publication}

No applicable.

\section{Availability of data and materials}

The datasets presented in this study can be found in online repositories. The names of the repository/repositories and accession number(s) can be found in the article/Supplementary materials.

\section{Competing interests}

The authors declare that they have no known competing financial interests or personal relationships that could have appeared to influence the work reported in this paper. 


\section{Funding}

No funding.

\section{Authors' contributions}

Conception and design: Qiaonan Guo, Pengjun Qiu, Jianqing Lin. Development of methodology: Qiaonan Guo, Pengjun Qiu. Acquisition of data (databases acquiring and data processing, etc.): Qiaonan Guo, Pengjun Qiu. Analysis and interpretation of data (e.g., statistical analysis, biostatistics, computational analysis): Qiaonan Guo, Pengjun Qiu, Jianpeng Chen. Writing, review, and/or revision of the manuscript: Qiaonan Guo, Kelun Pan, Pengjun Qiu, Jianqing Lin. Administrative, technical, or material support (i.e., reporting or organizing data, constructing databases): Qiaonan Guo, Pengjun Qiu. Study supervision: Jianqing Lin. All authors reviewed the manuscript. The authors disclose no potential conflicts of interest.

\section{Acknowledgements}

The authors would like to thank all researchers contributed to the TCGA and GEO data sets included.

\section{References}

1. Siegel R, Miller K, Jemal A: Cancer statistics, 2020. CA Cancer J Clin 2020, 70(1):7-30.

2. Harbeck N, Penault-Llorca F, Cortes J, Gnant M, Houssami N, Poortmans P, Ruddy K, Tsang J, Cardoso F: Breast cancer. Nature reviews Disease primers 2019, 5(1):66.

3. Olver I: New initiatives in the treatment of breast cancer. The Medical journal of Australia 2016, 205(10):449-450.

4. Zhu L, Tian Q, Jiang S, Gao H, Yu S, Zhou Y, Yan Y, Ren Y, He J, Wang B: A Novel Ferroptosis-Related Gene Signature for Overall Survival Prediction in Patients With Breast Cancer. Frontiers in cell and developmental biology 2021, 9:670184.

5. Bergin A, Loi S: Triplenegative breast cancer. recent treatment advances. F1000Research 2019, 8.

6. Eiró N, Fernandez-Garcia B, González L, Vizoso F: Cytokines related to MMP-11 expression by inflammatory cells and breast cancer metastasis. Oncoimmunology 2013, 2(5):e24010.

7. Harao M, Forget M, Roszik J, Gao H, Babiera G, Krishnamurthy S, Chacon J, Li S, Mittendorf E, DeSnyder $S$ et al: 4-1BB-Enhanced Expansion of CD8 TIL from Triple-Negative Breast Cancer Unveils Mutation-Specific CD8 T Cells. Cancer immunology research 2017, 5(6):439-445.

8. Bieniasz-Krzywiec P, Martín-Pérez R, Ehling M, García-Caballero M, Pinioti S, Pretto S, Kroes R, Aldeni $\mathrm{C}$, Di Matteo M, Prenen $\mathrm{H}$ et al: Podoplanin-Expressing Macrophages Promote Lymphangiogenesis and Lymphoinvasion in Breast Cancer. Cell metabolism 2019, 30(5):917-936.e910. 
9. Michea P, Noël F, Zakine E, Czerwinska U, Sirven P, Abouzid O, Goudot C, Scholer-Dahirel A, VincentSalomon A, Reyal $\mathrm{F}$ et al: Adjustment of dendritic cells to the breast-cancer microenvironment is subset specific. Nat Immunol 2018, 19(8):885-897.

10. Kennedy L, Salama A: A review of cancer immunotherapy toxicity. CA Cancer J Clin 2020, 70(2):86104.

11. Hui L, Chen Y: Tumor microenvironment: Sanctuary of the devil. Cancer Lett 2015, 368(1):7-13.

12. Pan B, Teng K, Wu C, Adam M, Johnstone R: Electron microscopic evidence for externalization of the transferrin receptor in vesicular form in sheep reticulocytes. The Journal of cell biology 1985 , 101(3):942-948.

13. Yu B, Zhang X, Li X: Exosomes derived from mesenchymal stem cells. International journal of molecular sciences 2014, 15(3):4142-4157.

14. EL Andaloussi S, Mäger I, Breakefield X, Wood M: Extracellular vesicles: biology and emerging therapeutic opportunities. Nature reviews Drug discovery 2013, 12(5):347-357.

15. Huang Y, Liu K, Li Q, Yao Y, Wang Y: Exosomes Function in Tumor Immune Microenvironment. Adv Exp Med Bio/ 2018, 1056:109-122.

16. Chalmin F, Ladoire S, Mignot G, Vincent J, Bruchard M, Remy-Martin J, Boireau W, Rouleau A, Simon $B$, Lanneau D et al: Membrane-associated Hsp72 from tumor-derived exosomes mediates STAT3dependent immunosuppressive function of mouse and human myeloid-derived suppressor cells. The Journal of clinical investigation 2010, 120(2):457-471.

17. Xiang X, Poliakov A, Liu C, Liu Y, Deng Z, Wang J, Cheng Z, Shah S, Wang G, Zhang L et al: Induction of myeloid-derived suppressor cells by tumor exosomes. Int J Cancer 2009, 124(11):2621-2633.

18. Berchem G, Noman M, Bosseler M, Paggetti J, Baconnais S, Le Cam E, Nanbakhsh A, Moussay E, Mami-Chouaib F, Janji B et al: Hypoxic tumor-derived microvesicles negatively regulate NK cell function by a mechanism involving TGF- $\beta$ and miR23a transfer. Oncoimmunology 2016, 5(4):e1062968.

19. Bryant R, Pawlowski T, Catto J, Marsden G, Vessella R, Rhees B, Kuslich C, Visakorpi T, Hamdy F: Changes in circulating microRNA levels associated with prostate cancer. Br J Cancer 2012, 106(4):768-774.

20. Kim H, Song K, Park Y, Kang Y, Lee Y, Lee K, Kim H, Ryu K, Bae J, Kim S: Elevated levels of circulating platelet microparticles, VEGF, IL-6 and RANTES in patients with gastric cancer: possible role of a metastasis predictor. European journal of cancer (Oxford, England: 1990) 2003, 39(2):184-191.

21. Wu J, Shen Z: Exosomal miRNAs as biomarkers for diagnostic and prognostic in lung cancer. Cancer medicine 2020, 9(19):6909-6922.

22. Melo S, Sugimoto H, O'Connell J, Kato N, Villanueva A, Vidal A, Qiu L, Vitkin E, Perelman L, Melo C et al: Cancer exosomes perform cell-independent microRNA biogenesis and promote tumorigenesis. Cancer Cell 2014, 26(5):707-721.

23. Peinado H, Alečković M, Lavotshkin S, Matei I, Costa-Silva B, Moreno-Bueno G, Hergueta-Redondo M, Williams C, García-Santos G, Ghajar C et al: Melanoma exosomes educate bone marrow progenitor 
cells toward a pro-metastatic phenotype through MET. Nat Med 2012, 18(6):883-891.

24. Wang X, Chai Z, Pan G, Hao Y, Li B, Ye T, Li Y, Long F, Xia L, Liu M: ExoBCD: a comprehensive database for exosomal biomarker discovery in breast cancer. Briefings in bioinformatics 2021, 22(3).

25. Saleh R, Toor S, Khalaf S, Elkord E: Breast Cancer Cells and PD-1/PD-L1 Blockade Upregulate the Expression of PD-1, CTLA-4, TIM-3 and LAG-3 Immune Checkpoints in CD4 T Cells. Vaccines 2019, 7(4).

26. Wang $\mathrm{H}$, Lengerich $\mathrm{B}$, Aragam $\mathrm{B}$, Xing E: Precision Lasso: accounting for correlations and linear dependencies in high-dimensional genomic data. Bioinformatics (Oxford, England) 2019, 35(7):1181-1187.

27. Yoshihara K, Shahmoradgoli M, Martínez E, Vegesna R, Kim H, Torres-Garcia W, Treviño V, Shen H, Laird P, Levine D et al: Inferring tumour purity and stromal and immune cell admixture from expression data. Nature communications 2013, 4:2612.

28. Newman A, Liu C, Green M, Gentles A, Feng W, Xu Y, Hoang C, Diehn M, Alizadeh A: Robust enumeration of cell subsets from tissue expression profiles. Nature methods 2015, 12(5):453-457.

29. Sousa B, Ribeiro A, Paredes J: Heterogeneity and Plasticity of Breast Cancer Stem Cells. Adv Exp Med Biol 2019, 1139:83-103.

30. Wu C, Du S, Zhang J, Liang A, Liu Y: Exosomes and breast cancer: a comprehensive review of novel therapeutic strategies from diagnosis to treatment. Cancer Gene Ther 2017, 24(1):6-12.

31. Chen Y, Zhang Y, Lv J, Li Y, Wang Y, He Q, Yang X, Sun Y, Mao Y, Yun J et al: Genomic Analysis of Tumor Microenvironment Immune Types across 14 Solid Cancer Types: Immunotherapeutic Implications. Theranostics 2017, 7(14):3585-3594.

32. Melo S, Luecke L, Kahlert C, Fernandez A, Gammon S, Kaye J, LeBleu V, Mittendorf E, Weitz J, Rahbari $\mathrm{N}$ et al: Glypican-1 identifies cancer exosomes and detects early pancreatic cancer. Nature 2015, 523(7559):177-182.

33. Chaudhary P, Gibbs L, Maji S, Lewis C, Suzuki S, Vishwanatha J: Serum exosomal-annexin A2 is associated with African-American triple-negative breast cancer and promotes angiogenesis. Breast cancer research: BCR 2020, 22(1):11.

34. Osta W, Chen Y, Mikhitarian K, Mitas M, Salem M, Hannun Y, Cole D, Gillanders W: EpCAM is overexpressed in breast cancer and is a potential target for breast cancer gene therapy. Cancer Res 2004, 64(16):5818-5824.

35. Terris B, Cavard C, Perret C: EpCAM, a new marker for cancer stem cells in hepatocellular carcinoma. $J$ Hepatol 2010, 52(2):280-281.

36. Baumann P, Cremers N, Kroese F, Orend G, Chiquet-Ehrismann R, Uede T, Yagita H, Sleeman J: CD24 expression causes the acquisition of multiple cellular properties associated with tumor growth and metastasis. Cancer Res 2005, 65(23):10783-10793.

37. Senner V, Sturm A, Baur I, Schrell U, Distel L, Paulus W: CD24 promotes invasion of glioma cells in vivo. J Neuropathol Exp Neurol 1999, 58(8):795-802. 
38. Schabath H, Runz S, Joumaa S, Altevogt P: CD24 affects CXCR4 function in pre-B lymphocytes and breast carcinoma cells. J Cell Sci 2006, 119:314-325.

39. Smith S, Oxford G, Wu Z, Nitz M, Conaway M, Frierson H, Hampton G, Theodorescu D: The metastasis-associated gene CD24 is regulated by Ral GTPase and is a mediator of cell proliferation and survival in human cancer. Cancer Res 2006, 66(4):1917-1922.

40. Wang W, Wang X, Peng L, Deng Q, Liang Y, Qing H, Jiang B: CD24-dependent MAPK pathway activation is required for colorectal cancer cell proliferation. Cancer Sci 2010, 101(1):112-119.

41. Yin S, Gao F: Molecular Mechanism of Tumor Cell Immune Escape Mediated by CD24/Siglec-10. Front Immunol 2020, 11:1324.

42. Barkal A, Brewer R, Markovic M, Kowarsky M, Barkal S, Zaro B, Krishnan V, Hatakeyama J, Dorigo O, Barkal $\mathrm{L}$ et al: CD24 signalling through macrophage Siglec-10 is a target for cancer immunotherapy. Nature 2019, 572(7769):392-396.

43. Qi X, Li X, Sun X: Reduced expression of polymeric immunoglobulin receptor (plgR) in nasopharyngeal carcinoma and its correlation with prognosis. Tumour biology: the journal of the International Society for Oncodevelopmental Biology and Medicine 2016, 37(8):11099-11104.

44. Su J, Miao L, YeX, Cui M, He X: Development of prognostic signature and nomogram for patients with breast cancer. Medicine 2019, 98(11):e14617.

45. Bracken J, Ghanem T, Kasem A, Jiang WG, Mokbel K: Evidence for Tumour Suppressor Function of DOK7 in Human Breast Cancer. J Cancer Ther 2014, 05(01):67-73.

46. Yue C, Bai Y, Piao Y, Liu H: DOK7 Inhibits Cell Proliferation, Migration, and Invasion of Breast Cancer via the PI3K/PTEN/AKT Pathway. J Oncol 2021, 2021:4035257.

47. Zheng Z, Cai Y, Chen H, Chen Z, Zhu D, Zhong Q, Xie W: CXCL13/CXCR5 Axis Predicts Poor Prognosis and Promotes Progression Through PI3K/AKT/mTOR Pathway in Clear Cell Renal Cell Carcinoma. Front Oncol 2018, 8:682.

48. Li C, Kang D, Sun X, Liu Y, Wang J, Gao P: The Effect of C-X-C Motif Chemokine 13 on Hepatocellular Carcinoma Associates with Wnt Signaling. BioMed research international 2015, 2015:345413.

49. Rubio A, Porter T, Zhong X: Duality of B Cell-CXCL13 Axis in Tumor Immunology. Front Immunol 2020, 11:521110.

50. Song I, Heo S, Bang W, Park H, Park I, Kim Y, Park S, Roh J, Gong G, Lee H: Predictive Value of Tertiary Lymphoid Structures Assessed by High Endothelial Venule Counts in the Neoadjuvant Setting of Triple-Negative Breast Cancer. Cancer Res Treat 2017, 49(2):399-407.

51. Rachidi S, Qin T, Sun S, Zheng W, Li Z: Molecular profiling of multiple human cancers defines an inflammatory cancer-associated molecular pattern and uncovers KPNA2 as a uniform poor prognostic cancer marker. PLoS One 2013, 8(3):e57911.

52. Raposo G, Nijman H, Stoorvogel W, Liejendekker R, Harding C, Melief C, Geuze H: B lymphocytes secrete antigen-presenting vesicles. The Journal of experimental medicine 1996, 183(3):1161-1172. 
53. Zitvogel L, Regnault A, Lozier A, Wolfers J, Flament C, Tenza D, Ricciardi-Castagnoli P, Raposo G, Amigorena S: Eradication of established murine tumors using a novel cell-free vaccine: dendritic cellderived exosomes. Nat Med 1998, 4(5):594-600.

54. Bobrie A, Colombo M, Raposo G, Théry C: Exosome secretion: molecular mechanisms and roles in immune responses. Traffic (Copenhagen, Denmark) 2011, 12(12):1659-1668.

55. Gastpar R, Gehrmann M, Bausero M, Asea A, Gross C, Schroeder J, Multhoff G: Heat shock protein 70 surface-positive tumor exosomes stimulate migratory and cytolytic activity of natural killer cells. Cancer Res 2005, 65(12):5238-5247.

56. Andreola G, Rivoltini L, Castelli C, Huber V, Perego P, Deho P, Squarcina P, Accornero P, Lozupone F, Lugini $L$ et al: Induction of lymphocyte apoptosis by tumor cell secretion of FasL-bearing microvesicles. The Journal of experimental medicine 2002, 195(10):1303-1316.

57. Taylor D, Gerçel-Taylor C, Lyons K, Stanson J, Whiteside T: T-cell apoptosis and suppression of T-cell receptor/CD3-zeta by Fas ligand-containing membrane vesicles shed from ovarian tumors. Clinical cancer research: an official journal of the American Association for Cancer Research 2003, 9(14):5113-5119.

58. Clayton A, Mitchell J, Court J, Linnane S, Mason M, Tabi Z: Human tumor-derived exosomes downmodulate NKG2D expression. Journal of immunology (Baltimore, Md: 1950) 2008, 180(11):72497258.

59. Szajnik M, Czystowska M, Szczepanski M, Mandapathil M, Whiteside T: Tumor-derived microvesicles induce, expand and up-regulate biological activities of human regulatory T cells (Treg). PLOS One 2010, 5(7):e11469.

60. Valenti R, Huber V, Filipazzi P, Pilla L, Sovena G, Villa A, Corbelli A, Fais S, Parmiani G, Rivoltini L: Human tumor-released microvesicles promote the differentiation of myeloid cells with transforming growth factor-beta-mediated suppressive activity on T lymphocytes. Cancer Res 2006, 66(18):92909298.

61. Hanahan D, Weinberg R: Hallmarks of cancer: the next generation. Cel/ 2011, 144(5):646-674.

62. Mincheva-Nilsson L, Baranov V: Cancer exosomes and NKG2D receptor-ligand interactions: impairing NKG2D-mediated cytotoxicity and anti-tumour immune surveillance. Semin Cancer Bio/ 2014, 28:2430 .

63. Lv L, Wan Y, Lin Y, Zhang W, Yang M, Li G, Lin H, Shang C, Chen Y, Min J: Anticancer drugs cause release of exosomes with heat shock proteins from human hepatocellular carcinoma cells that elicit effective natural killer cell antitumor responses in vitro. The Journal of biological chemistry 2012, 287(19):15874-15885.

64. Yu S, Liu C, Su K, Wang J, Liu Y, Zhang L, Li C, Cong Y, Kimberly R, Grizzle W et al: Tumor exosomes inhibit differentiation of bone marrow dendritic cells. Journal of immunology (Baltimore, Md: 1950) 2007, 178(11):6867-6875.

65. Yang M, Chen J, Su F, Yu B, Su F, Lin L, Liu Y, Huang J, Song E: Microvesicles secreted by macrophages shuttle invasion-potentiating microRNAs into breast cancer cells. Mol Cancer 2011, 
10:117.

66. Bobrie A, Krumeich S, Reyal F, Recchi C, Moita L, Seabra M, Ostrowski M, Théry C: Rab27a supports exosomedependent and -independent mechanisms that modify the tumor microenvironment and can promote tumor progression. Cancer Res 2012, 72(19):4920-4930.

67. Bondhopadhyay B, Sisodiya S, Alzahrani F, Bakhrebah M, Chikara A, Kasherwal V, Khan A, Rani J, Dar S, Akhter N et al: Exosomes: A Forthcoming Era of Breast Cancer Therapeutics. Cancers (Basel) 2021, 13(18).

68. Yang Y, Li C, Chan L, Wei Y, Hsu J, Xia W, Cha J, Hou J, Hsu J, Sun L et al: Exosomal PD-L1 harbors active defense function to suppress $T$ cell killing of breast cancer cells and promote tumor growth. Cell Res 2018, 28(8):862-864.

\section{Figures}




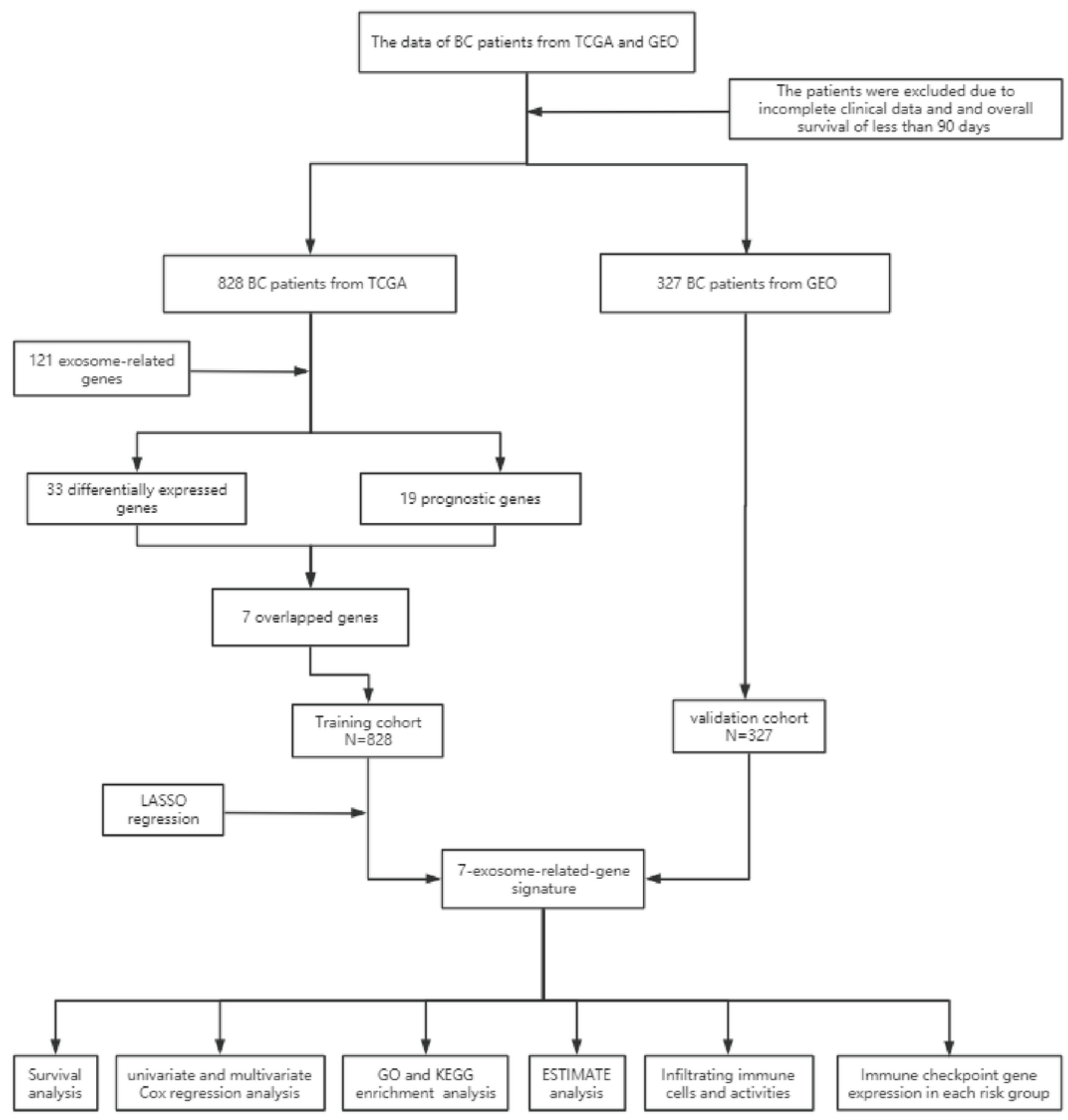

\section{Figure 1}

Analysis flow chart. 
A

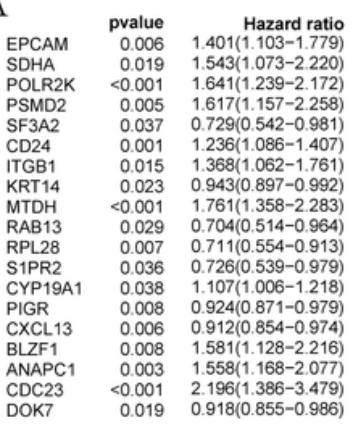

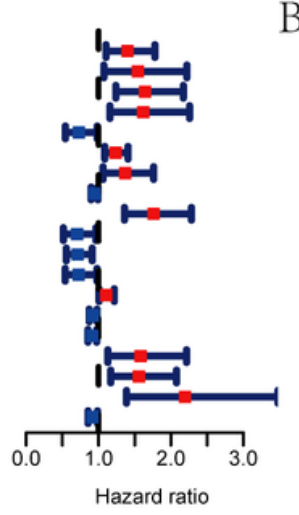

B

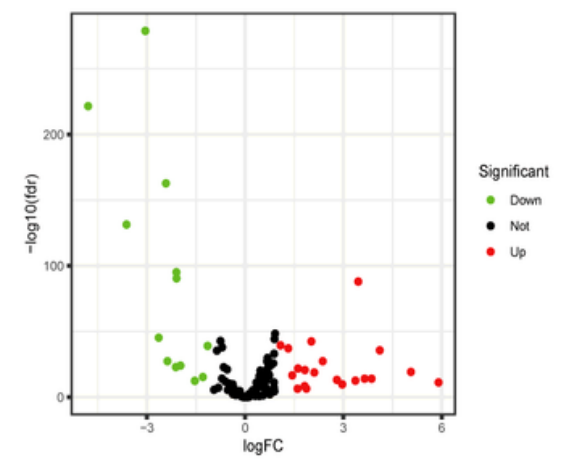

C

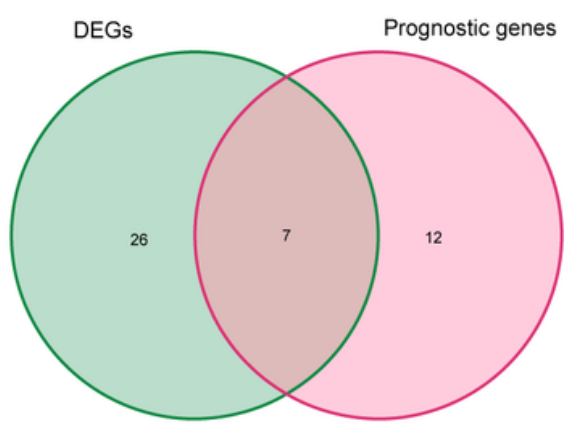

Figure 2

Identification of exosome-related genes differentially expressed in breast cancer patients with prognostic significance. (A) Identification of exosome-related genes with prognostic significance via univariate Cox regression analysis. 19 exosome-related genes were identified associated with prognosis in breast cancer patients $(\mathrm{P}<0.05)$. (B) The 33 overlapping genes differently expressed in normal and tumor tissue were identified and visualized by volcano map. (C) 7 overlapping genes between DEGs and prognostic exosome-related genes in the TCGA cohort were ascertained by Venn diagram.

A

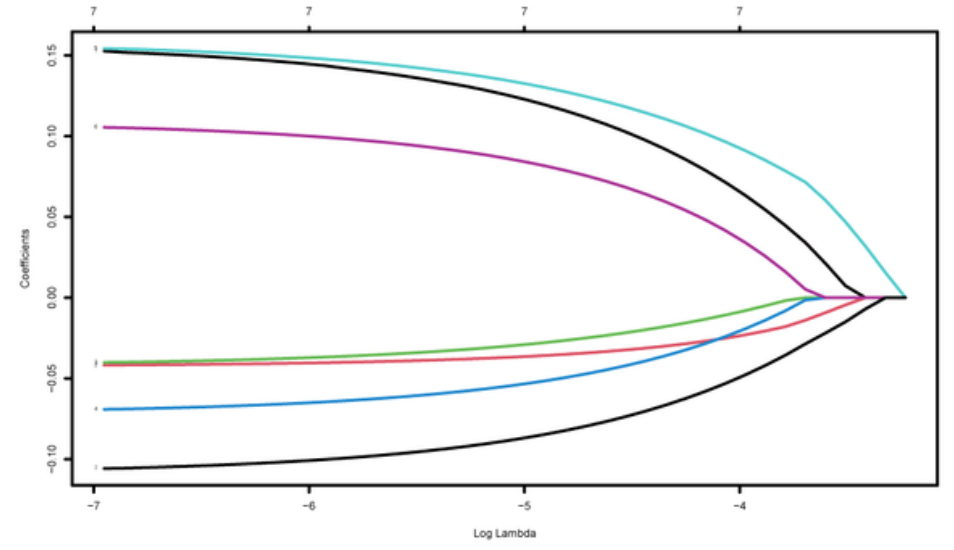

B

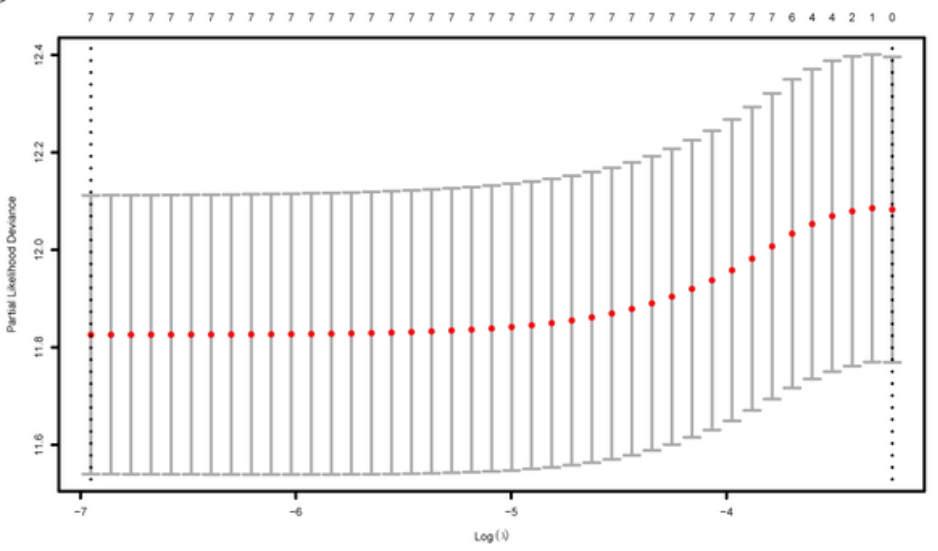

\section{Figure 3}

Identification of exosome-related genes in breast cancer patients with prognostic significance through LASSO Cox regression analysis. (A) LASSO coefficient profiles of 7 exosome-related genes with $P<0.01$. (B) The results of the 10 -fold cross-validation determined the optimal value of the penalty parameter $\lambda$. 
BC Patients from TCGA

A

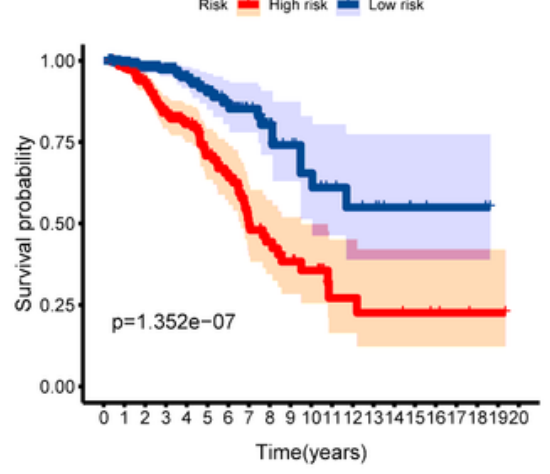

B

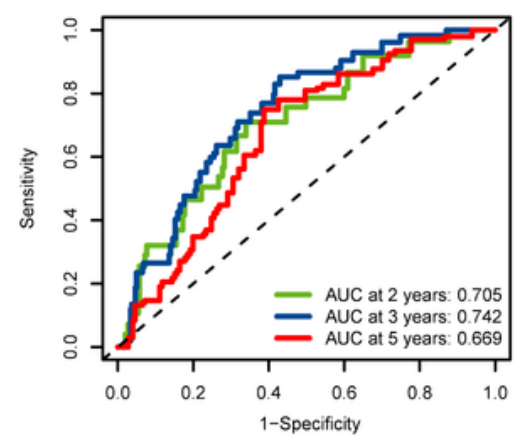

C

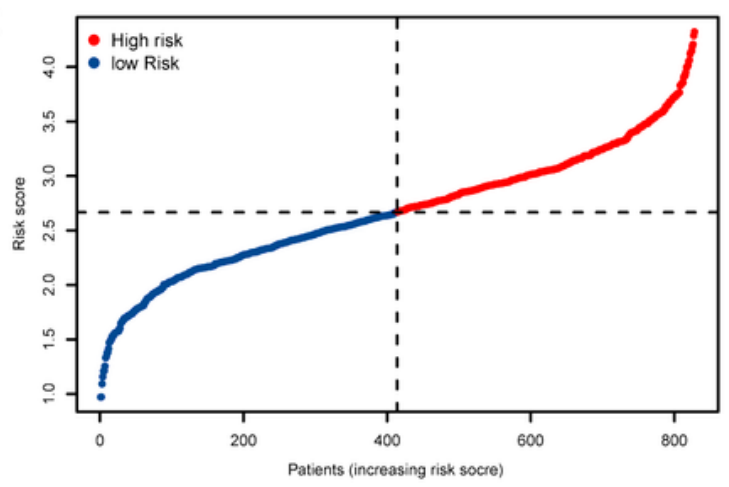

D

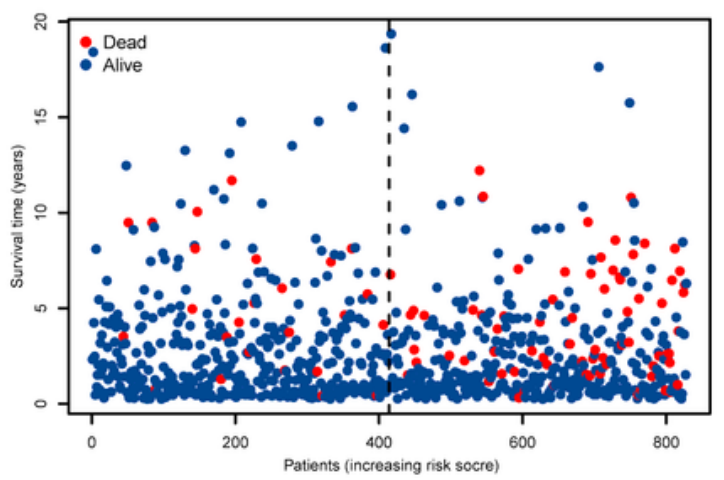

BC Patients from GEO

E

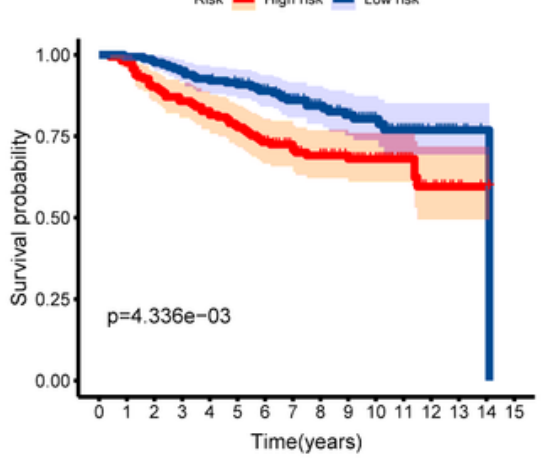

F

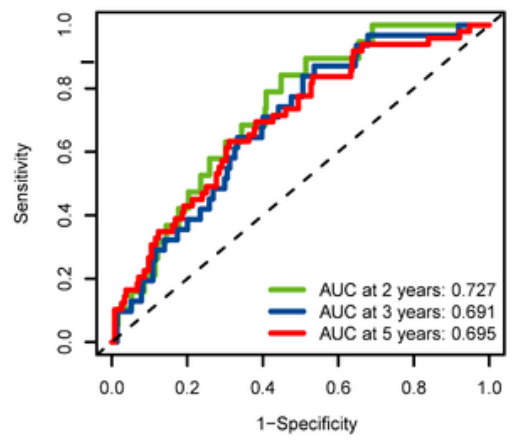

G

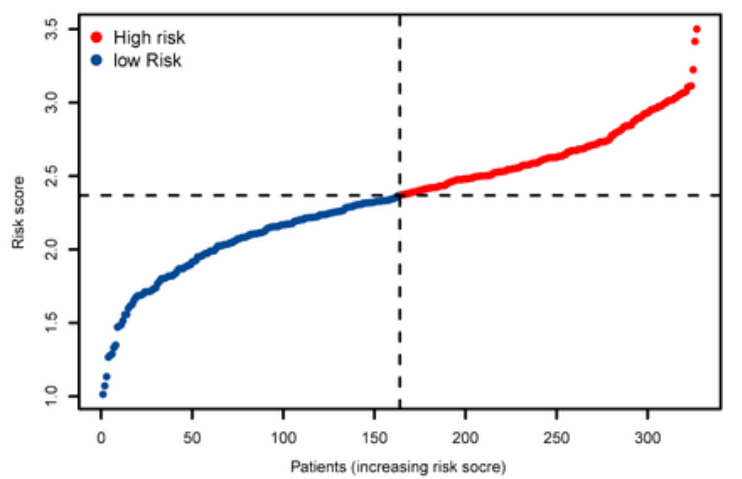

$\mathrm{H}$

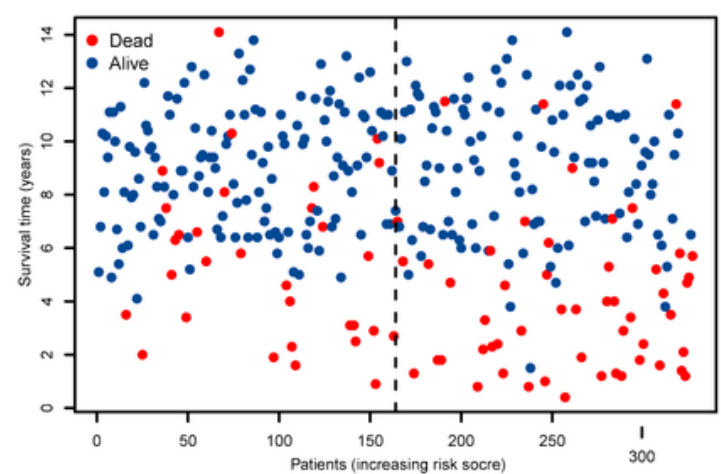

Figure 4

Efficacy and prognosis analysis of the exosome-associated risk model in the training and validation sets. (A, E) Kaplan-Meier survival curves for BC patients from TCGA cohort and GEO cohort, stratified based on risk scores (high vs. low); comparisons of the survival time in high-risk groups and low-risk groups with log-rank tests ( $p=1.3521 \mathrm{E}-07$ and $p=4.336 \mathrm{E}-03$, respectively). ROC curve analysis of model accuracy for predicting patient prognosis at 2, 3 and 5 years in the training (B) and validation (F) sets. 
The median value of the risk score in TCGA $(C)$ and GEO (G) cohorts. The distributions of survival status and risk scores in TCGA (D) and GEO $(H)$ cohorts.

\section{TCGA cohort}
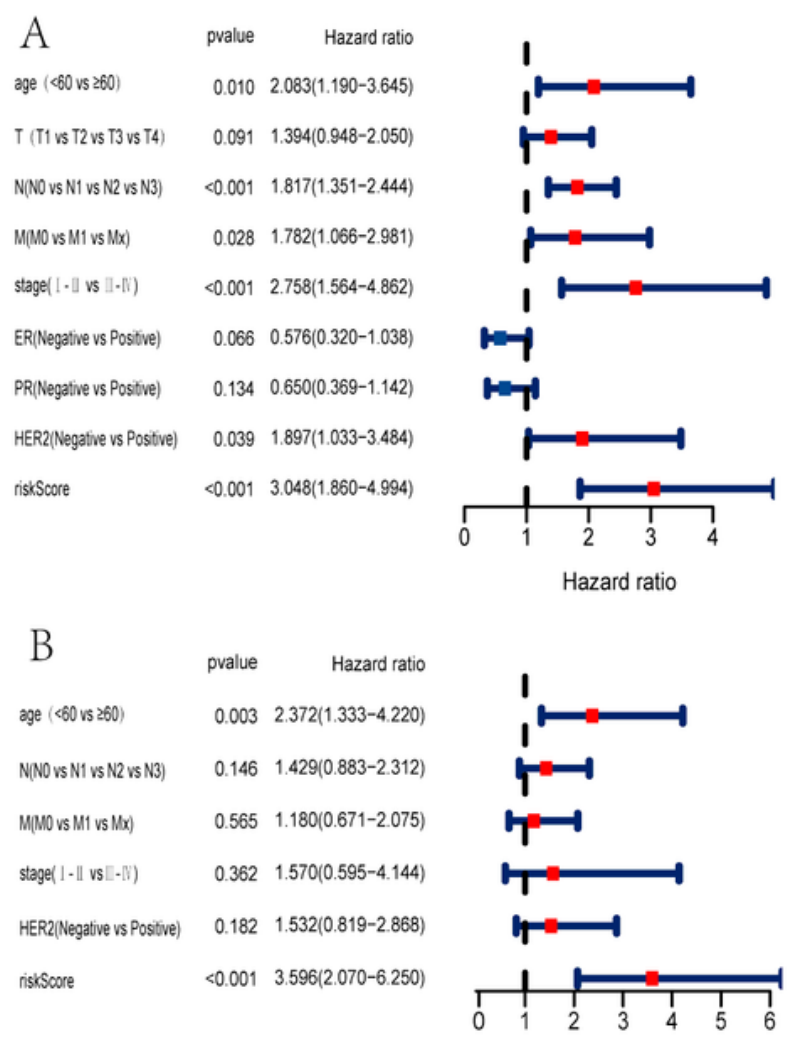

Hazard ratio

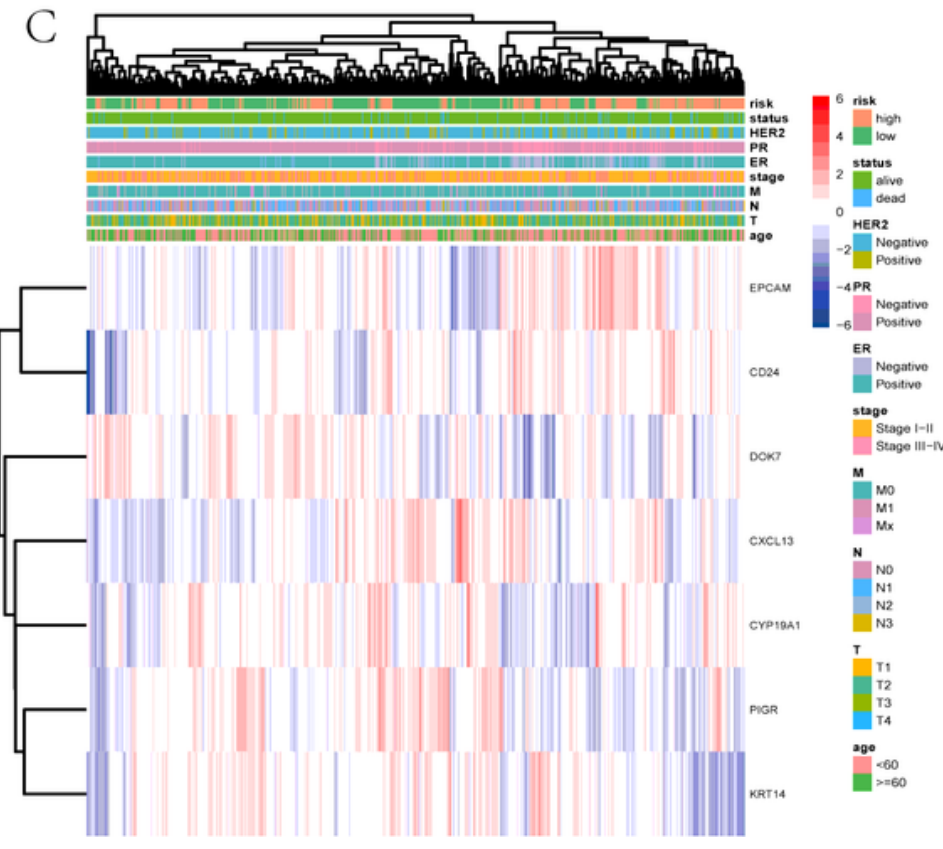

GEO cohort

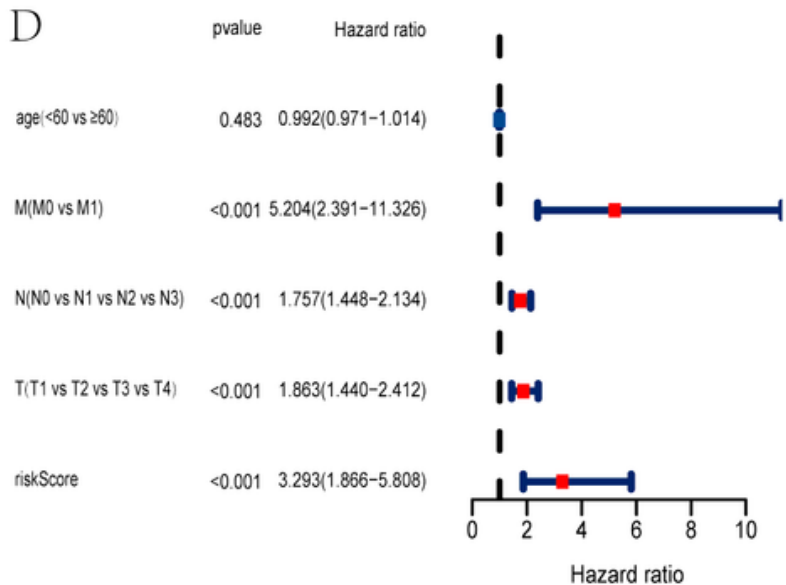

E
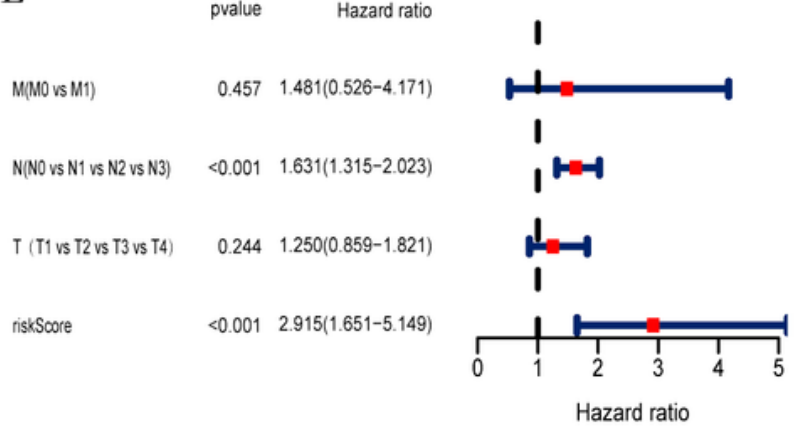

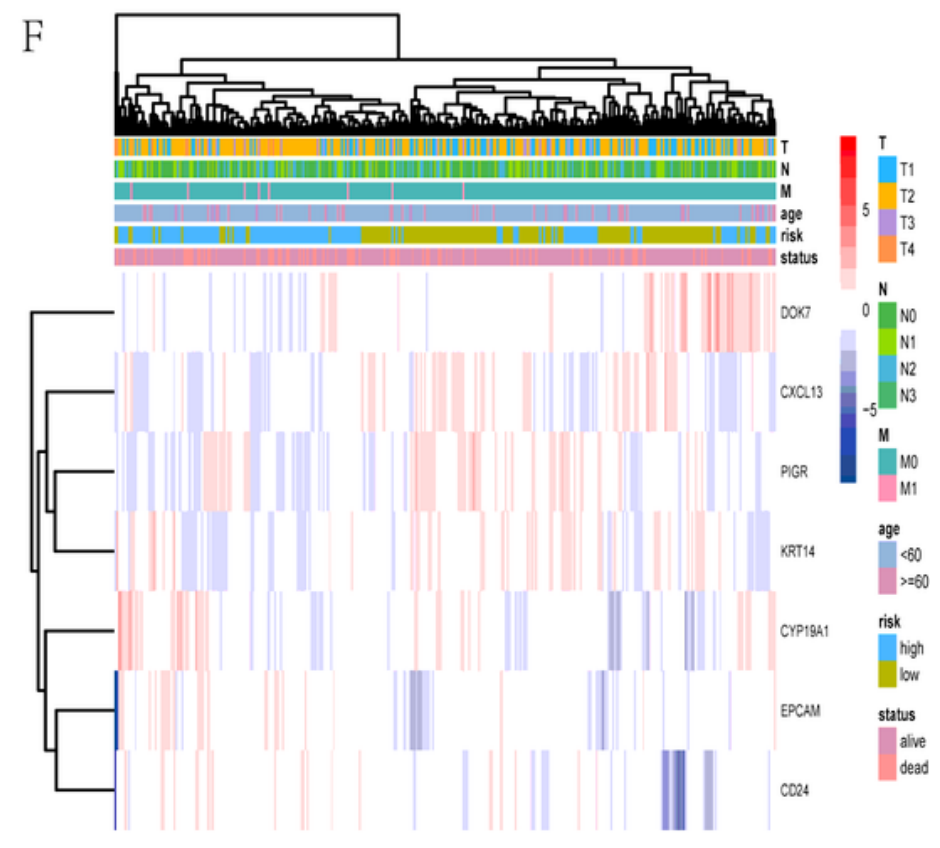

Figure 5

Independent prognostic value of the exosome-related risk model in the training and validation sets. The hierarchical clustering showed the associations between signature risk score, expression levels of 7 
exosome-related genes, and clinical features or molecular characteristics in the training and validation datasets. (A) The forest plots for univariate Cox regression analysis in TCGA cohort showed that risk

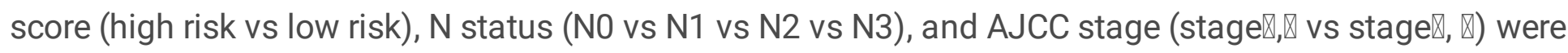
variables related to prognostic risk. (B) The forest plots for multivariate Cox regression analysis in TCGA cohort showed that risk score (high risk vs low risk) were independent prognostic factors. (D) The forest plots for univariate Cox regression analysis in GEO cohort showed that risk score (high risk vs low risk), $M$ status (M0 vs M1), N status (N0 vs N1 vs N2 vs N3), and T status (T1 vs T2 vs T3 vs T4) were variables related to prognostic risk. (E) The forest plots for multivariate Cox regression analysis in GEO cohort showed that risk score (high risk vs low risk), and N status (N0 vs N1 vs N2 vs N3) were independent prognostic factors. Heatmap illustrated the expression levels of 7 exosome-associated genes and molecular pathological characteristics or clinical features by use of hierarchical clustering in TCGA (C) and GEO (F) sets. 
complement activation, classical pathway
complement activation humoral immune response adaptive immune response. immunoglobulin mediated immune response. B cell receptor signaling pathway. B cell mediated immunity lymphocyte mediated immunity. immune response-activating cell surface receptor signaling pathway
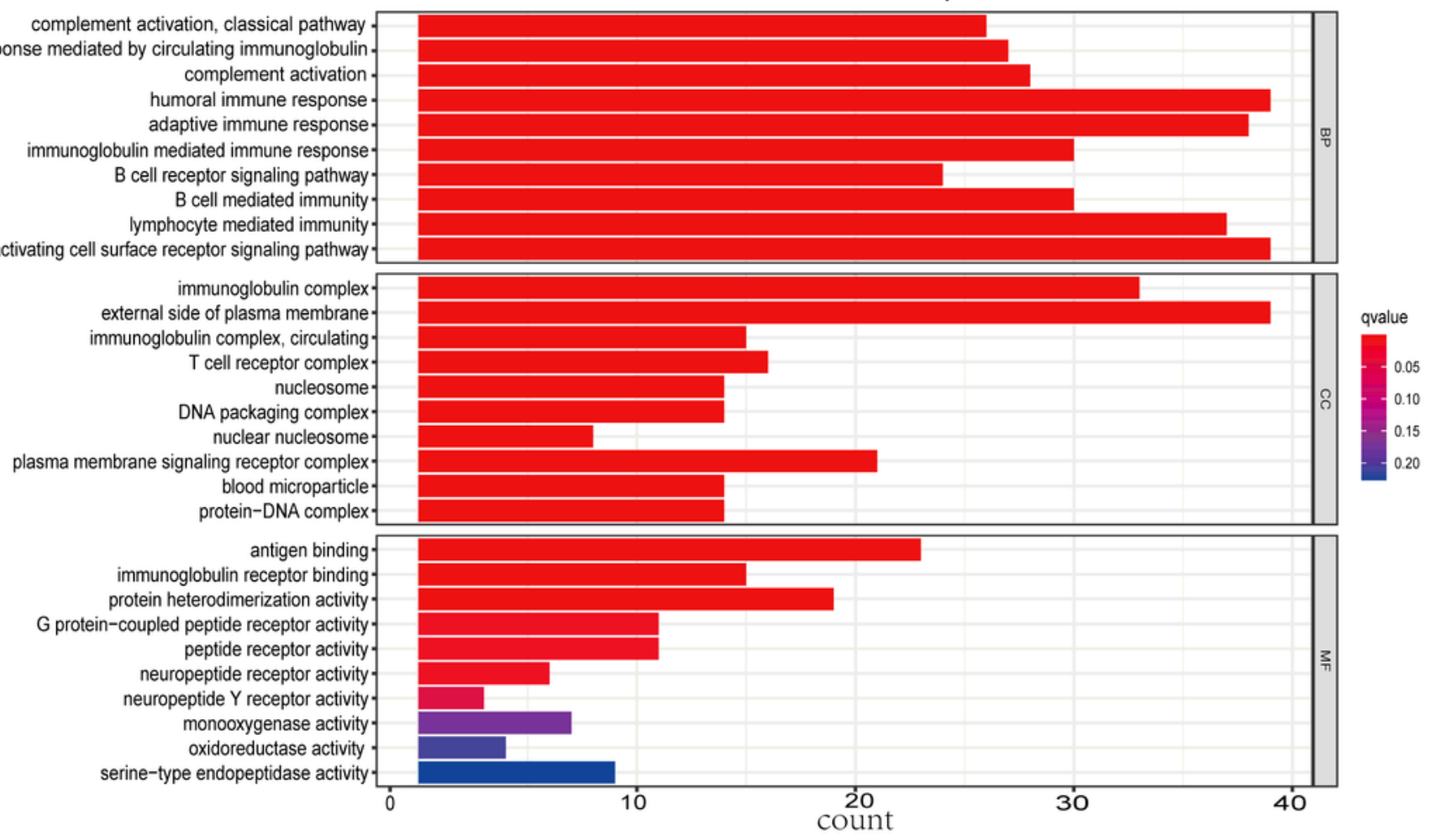

B

GEO cohort for the GO enrichment analysis

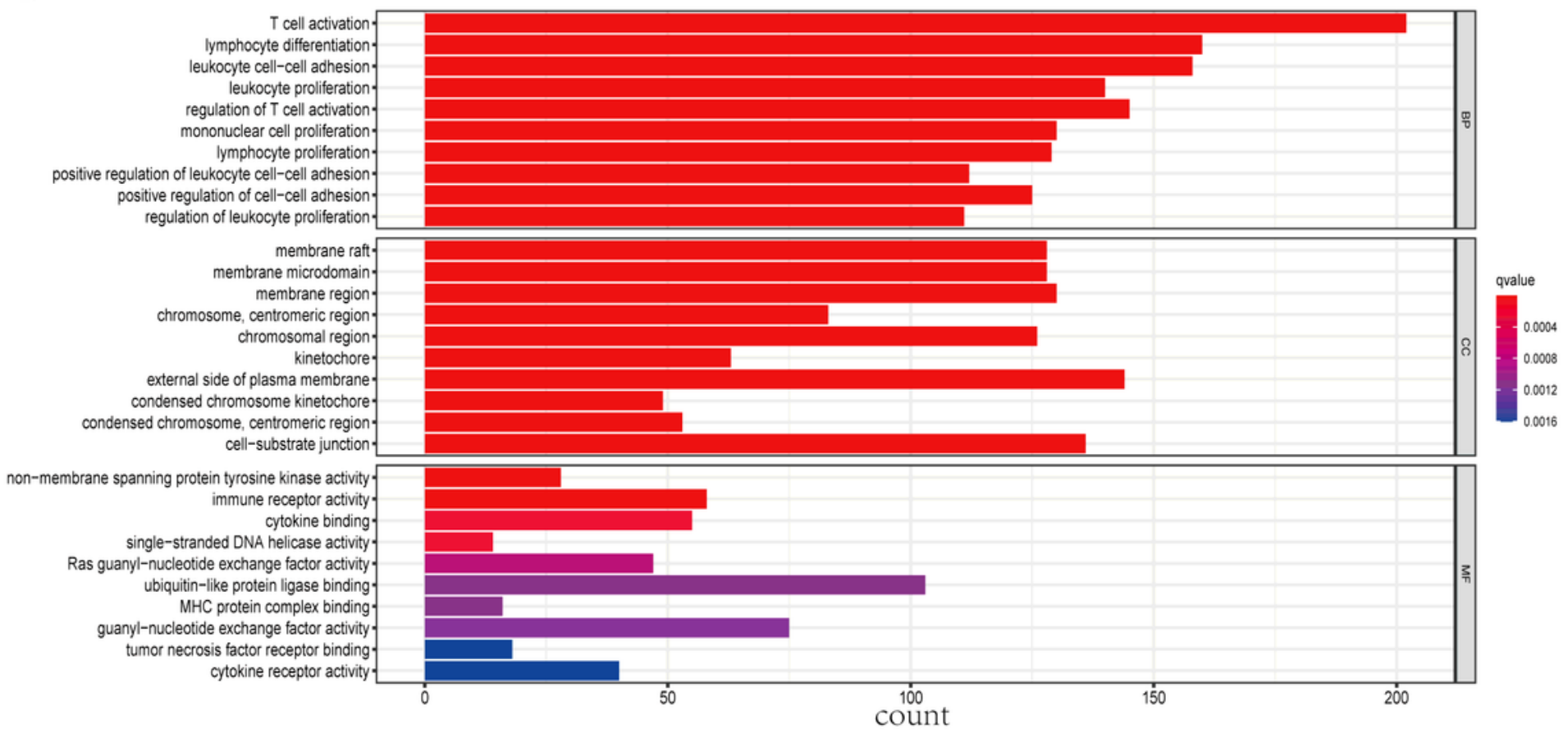

\section{Figure 6}

Representative findings from GO enrichment analyses in TCGA cohort and GEO cohort. The outcomes of biological process enrichment, cellular component enrichment, and molecular function enrichment in DEGs between high- and low- risk groups in TCGA database(A) and GEO database(B). 
A TCGA cohort for the KEGG analysis

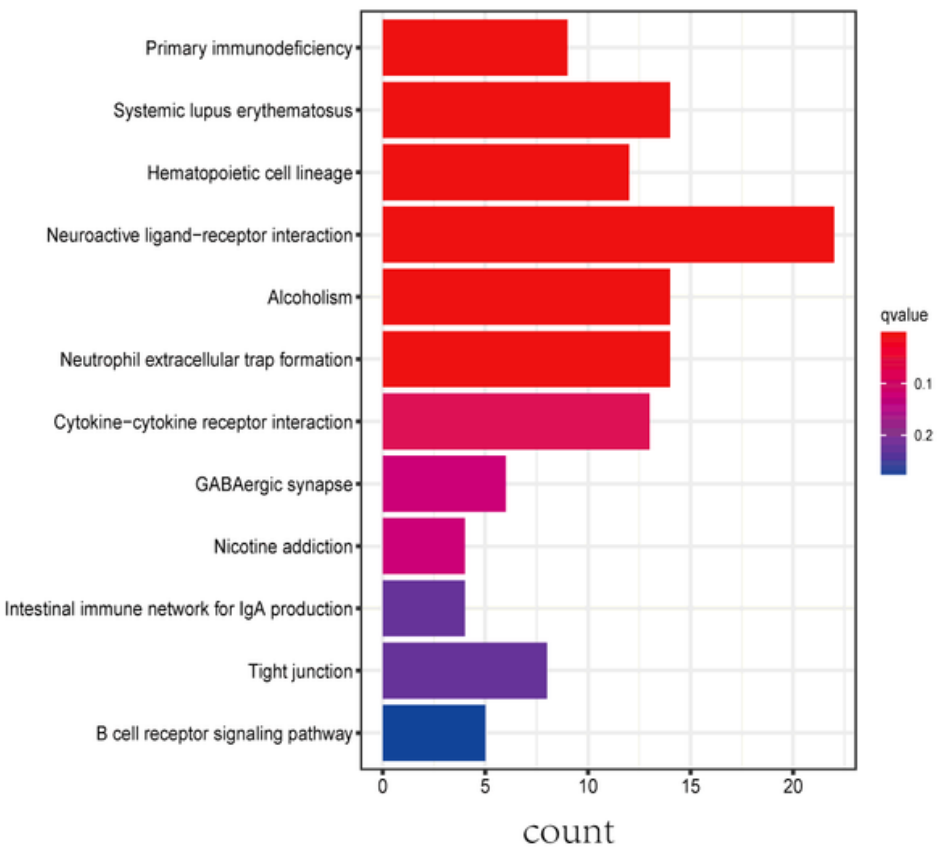

B GEO cohort for the KEGG analysis

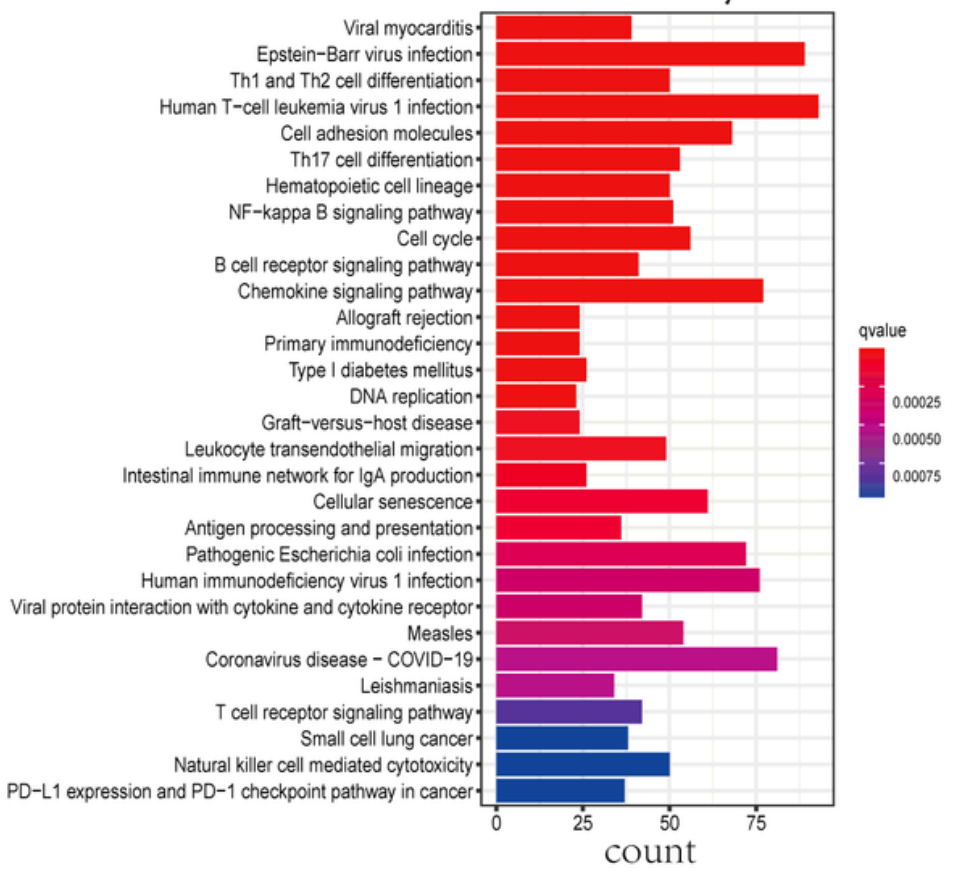

Figure 7

Representative findings from KEGG enrichment analyses in TCGA cohort and GEO cohort. The outcomes of KEGG pathways analyses in DEGs between high- and low- risk groups in TCGA database(A) and GEO database(B). 
A

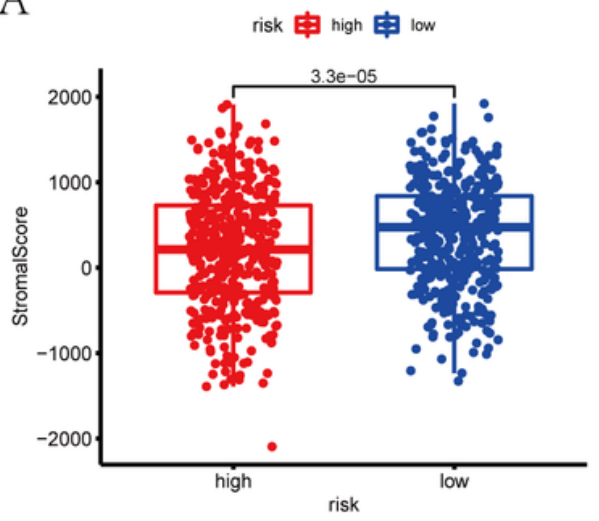

$D$

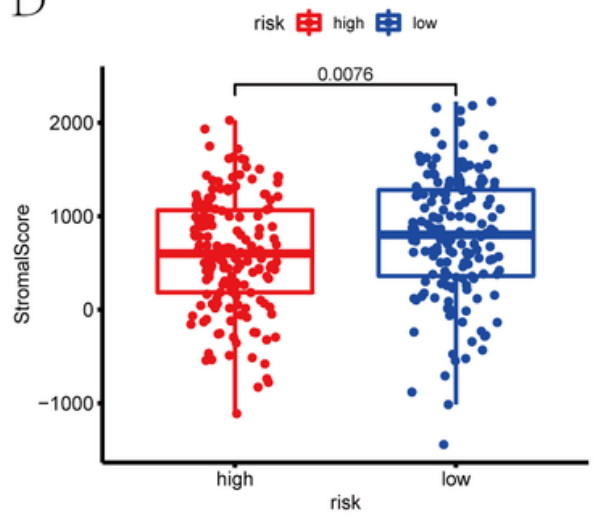

B

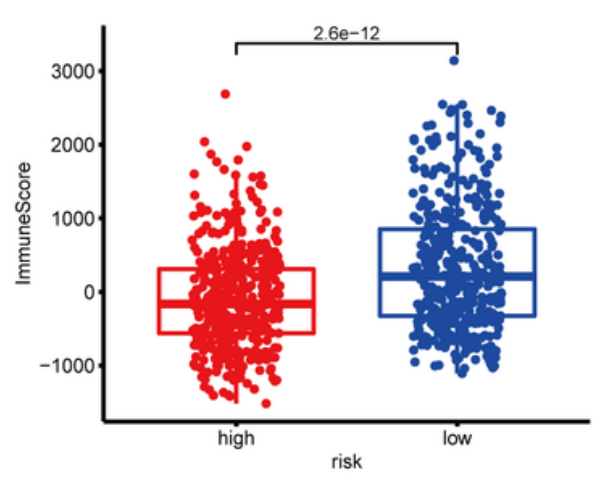

E

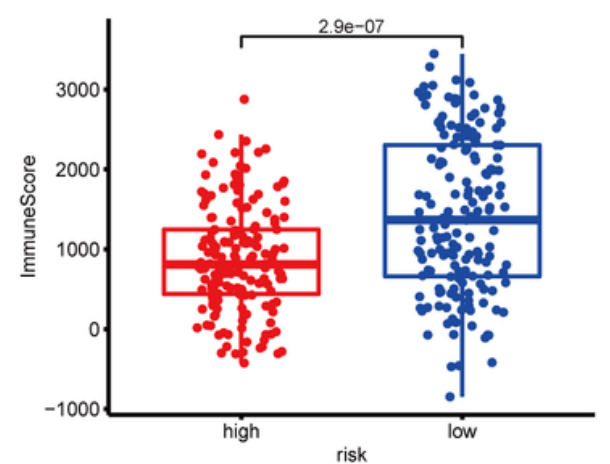

C risk 由 high 由 low

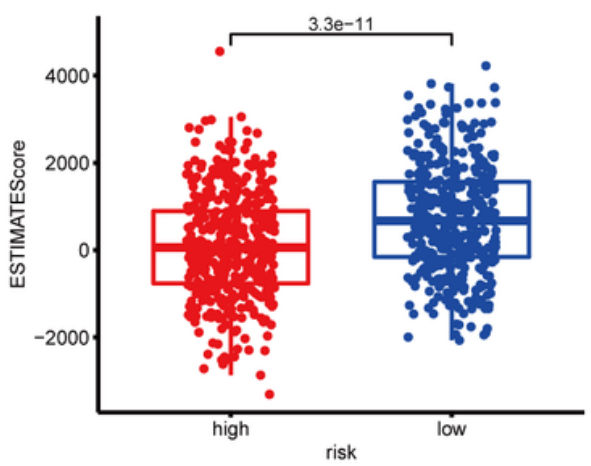

F risk 由 high 由 low

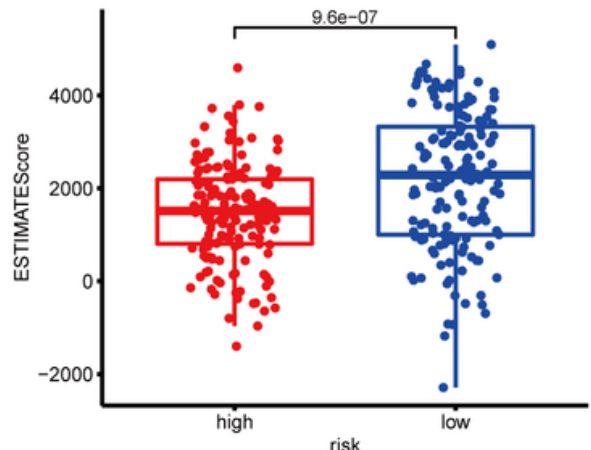

Figure 8

The scatter plots reveal that the distribution of stromal score, immune score and ESTIMATE score was different between high- and low- risk groups in the TCGA cohort (A, B and C) and GEO cohort (D, E and F). 


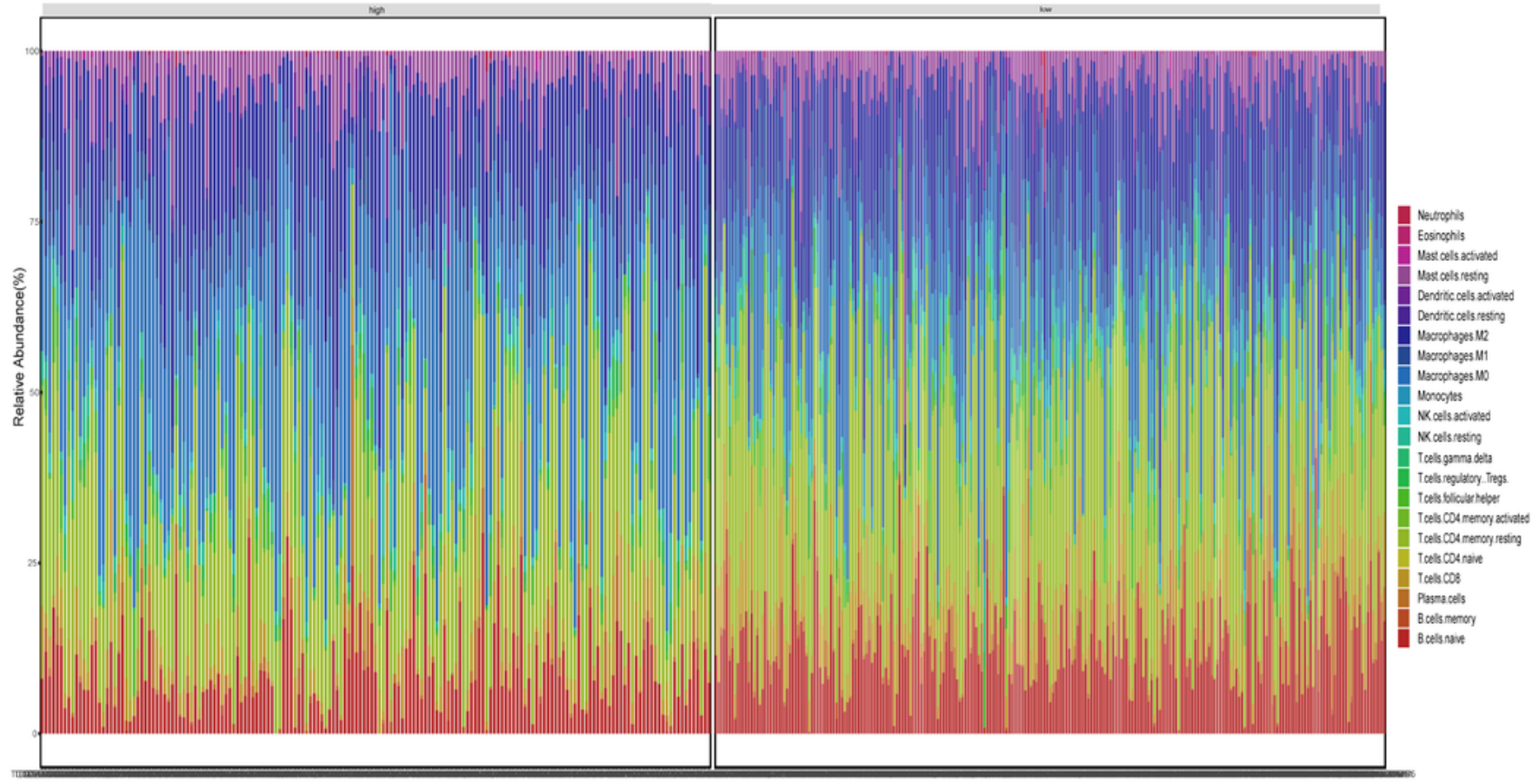

B

GEO cohort

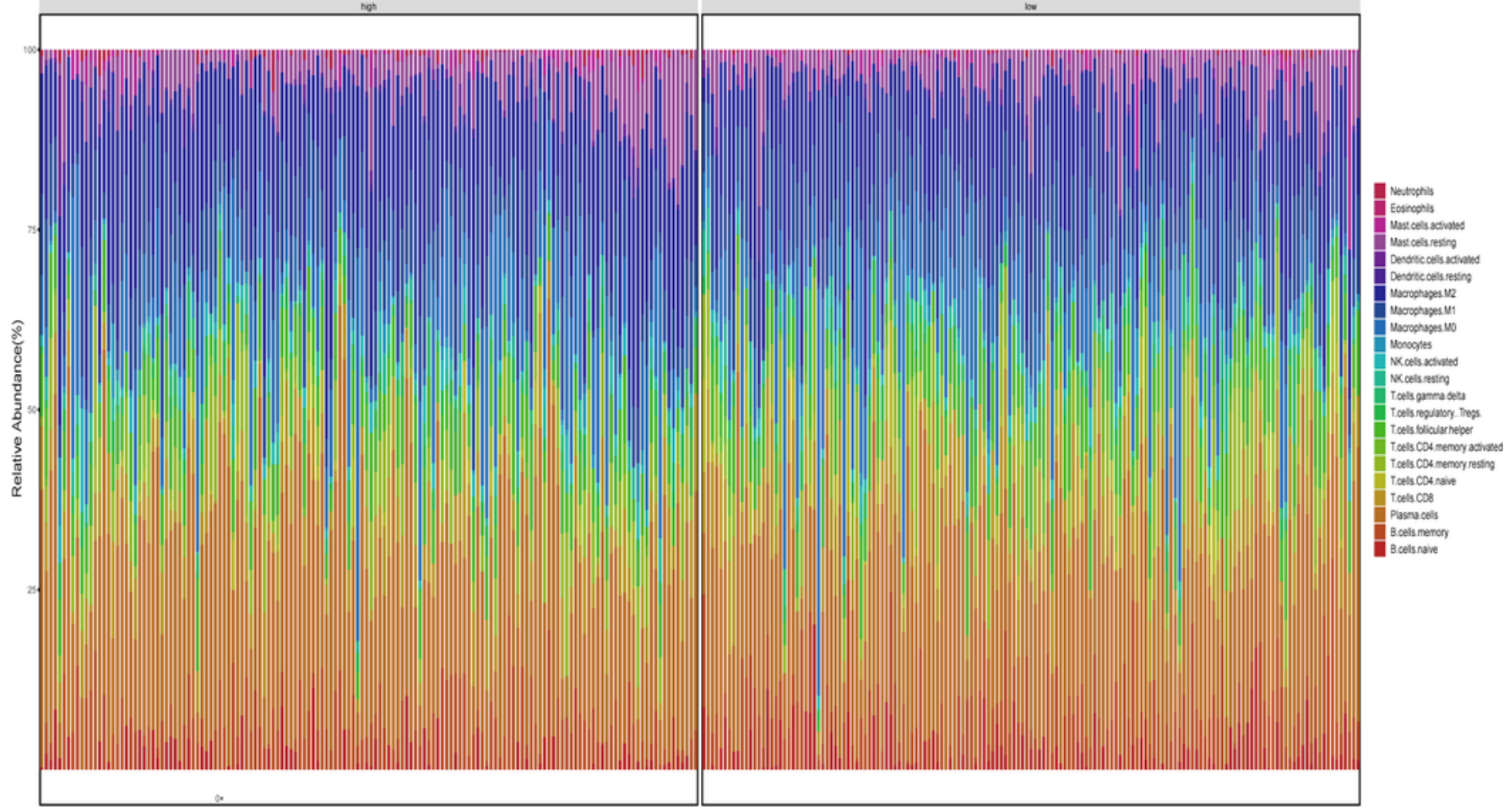

Figure 9

Immune infiltrations of TCGA and GEO cohort. Barplot showed the relative proportion of immune infiltration between high- and low- risk groups in TCGA cohort (A) and GEO cohort (B). 

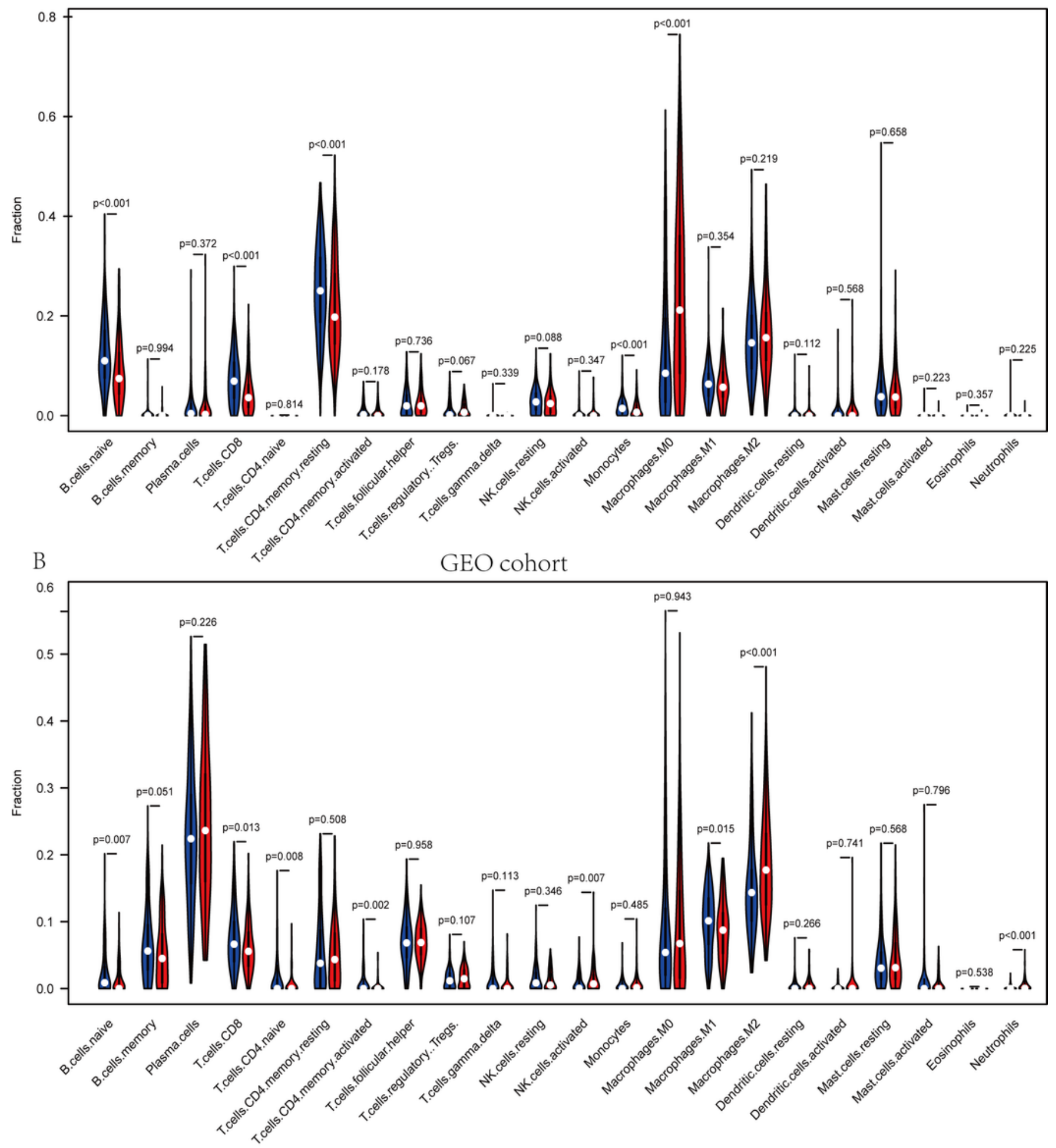

Figure 10

Apparent association of different immune cells between high- and low- risk groups in the TCGA cohort (A) and GEO cohort (B). 
TCGA cohort
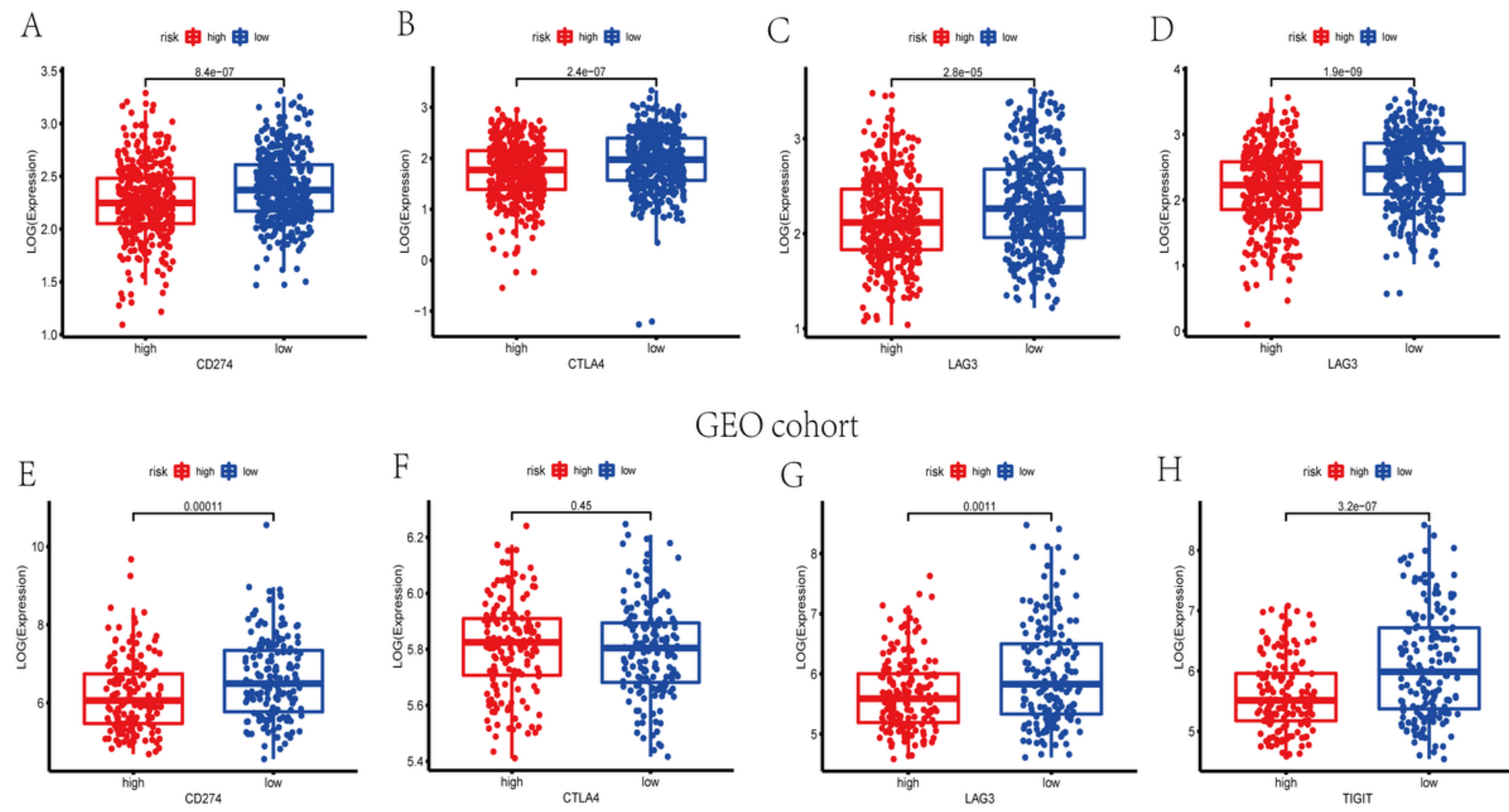

GEO cohort
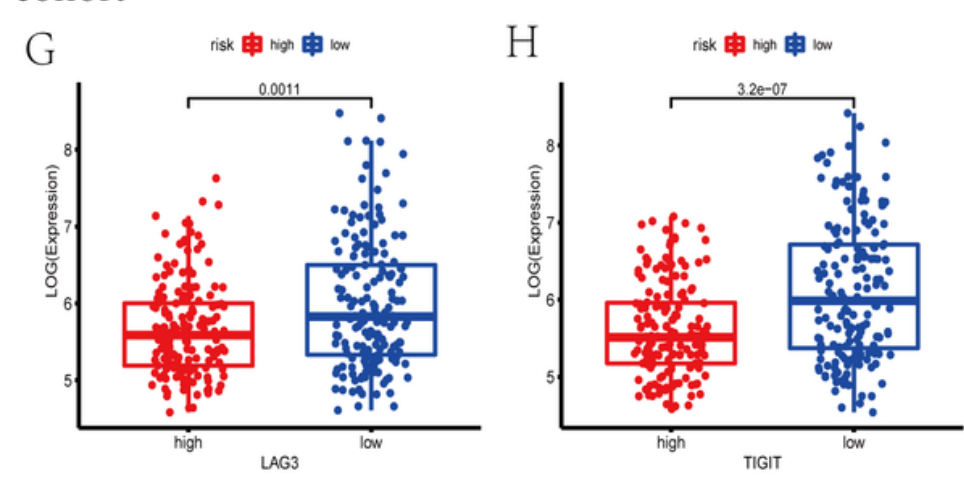

Figure 11

The expression levels of immune checkpoint genes in different risk groups. The expression levels of CD274 (A), CTLA4 (B), LAG3 (C) and TIGIT (D) in different risk groups of TCGA set $(P<0.001)$. The expression levels of CD274 (E), CTLA4 (F), LAG3 (G) and TIGIT $(H)$ in different risk groups of GEO set (CD274, LAG3 and TIGIT: $P<0.001 ;$ CTLA4: $P=0.45$ ).

\section{Supplementary Files}

This is a list of supplementary files associated with this preprint. Click to download.

- Supplementarytable1.csv 